\title{
Multiple intersection exponents for planar Brownian motion
}

\author{
Achim Klenke \\ Johannes Gutenberg-Universität Mainz \\ Institut für Mathematik \\ Staudingerweg 9 \\ 55099 Mainz \\ Germany \\ math@aklenke.de \\ http://www.aklenke.de \\ Tel. $+49+6131+3922829$ \\ Fax $+49+6131+3920916$
}

\author{
Peter Mörters \\ University of Bath \\ Department of Mathematical Sciences \\ Claverton Down \\ Bath BA2 7AY \\ United Kingdom \\ maspm@bath.ac.uk \\ http://www.bath.ac.uk/ maspm
}

November 29th, 2008, revised June 1st, 2009

\begin{abstract}
Let $p \geq 2, n_{1} \leq \cdots \leq n_{p}$ be positive integers and $B_{1}^{1}, \ldots, B_{n_{1}}^{1} ; \ldots ; B_{1}^{p}, \ldots, B_{n_{p}}^{p}$ be independent planar Brownian motions started uniformly on the boundary of the unit circle. We define a $p$-fold intersection exponent $\varsigma_{p}\left(n_{1}, \ldots, n_{p}\right)$, as the exponential rate of decay of the probability that the packets $\bigcup_{j=1}^{n_{i}} B_{j}^{i}\left[0, t^{2}\right], i=1, \ldots, p$, have no joint intersection. The case $p=2$ is well-known and, following two decades of numerical and mathematical activity, Lawler, Schramm and Werner (2001) rigorously identified precise values for these exponents. The exponents have not been investigated so far for $p>2$. We present an extensive mathematical and numerical study, leading to an exact formula in the case $n_{1}=1, n_{2}=2$, and several interesting conjectures for other cases.
\end{abstract}

AMS 2000 subject classification: $60 \mathrm{~J} 65$.

Keywords: Brownian motion, intersection exponent. 


\section{Introduction}

\subsection{Motivation and overview}

Finding exponents, which describe the decay of some probabilities, and dimensions of some sets associated with stochastic models of physical systems is one of the core activities in statistical physics. While in general one often has to resort to numerical methods to get a handle on the values of the exponents, for planar models conformal invariance may help to answer these questions explicitly, and there is now a substantial body of rigorous and non-rigorous methods available. For example, by making the assumption that critical planar percolation behaves in a conformally invariant way in the scaling limit and using ideas involving conformal field theory, Cardy [4] determined the asymptotic probability, as $N \rightarrow \infty$, that there exists a two-dimensional critical percolation cluster crossing a rectangle. A rigorous proof of Cardy's formula was later given by Smirnov [25]. Following considerable numerical work, see for example [18, 26] and references therein, Saleur and Duplantier [24] predicted the fractal dimension of the hull of a large percolation cluster using a non-rigorous Coulomb gas technique. Rigorous versions of this result have been given based on Cardy's formula, for example by Camia and Newman $[2,3]$.

In [6] Duplantier and Kwon suggested that ideas of conformal field theory can also be used to predict the probability of pairwise non-intersection between planar Brownian paths. Early research by Burdzy, Lawler and Polaski [1] and Li and Sokal [20] was of numerical nature, but ten years later, Duplantier [5] gave a derivation based on non-rigorous methods of quantum gravity, and soon after that Lawler, Schramm and Werner $[14,15,16]$ gave a rigorous proof based on the Schramm-Loewner evolution (SLE), one of the greatest achievements in probability in recent years. We also mention here some very recent developments with the long term aim of making the quantum gravity approach rigorous, see Duplantier and Sheffield [7], and Rhodes and Vargas [23].

In this paper we look at joint intersections of three or more planar Brownian paths, a question which has been neglected so far in the literature, but which came up in our recent investigation of the multifractality of intersection local times [8]. In the simplest case, given three independent Brownian paths $B^{1}, B^{2}, B^{3}$ started uniformly on the unit circle, we are interested in the asymptotic behaviour, as $t \rightarrow \infty$, of the non-intersection probability

$$
\mathbb{P}\left\{B^{1}[0, t] \cap B^{2}[0, t] \cap B^{3}[0, t]=\emptyset\right\} .
$$

Observe that this probability goes to zero, for $t \uparrow \infty$, as three, or any finite number, of Brownian paths in the plane eventually intersect, see e.g. [21, Chapter 9.1]. Recall for comparison, that the non-intersection exponents for three Brownian paths studied in the aforementioned papers deal with pairwise non-intersections, i.e. in the case of three Brownian motions either with

$$
\begin{aligned}
\mathbb{P}\left\{B^{1}[0, t] \cap B^{2}[0, t]=\right. & \left.\emptyset, B^{2}[0, t] \cap B^{3}[0, t]=\emptyset, B^{1}[0, t] \cap B^{3}[0, t]=\emptyset\right\}, \quad \text { or with } \\
& \mathbb{P}\left\{B^{1}[0, t] \cap\left(B^{2}[0, t] \cup B^{3}[0, t]\right)=\emptyset\right\} .
\end{aligned}
$$

Our study starts with the observation that, for positive integers $n_{1}, \ldots, n_{p}$ and independent planar Brownian motions

$$
B_{1}^{1}, \ldots, B_{n_{1}}^{1} ; \ldots ; B_{1}^{p}, \ldots, B_{n_{p}}^{p},
$$




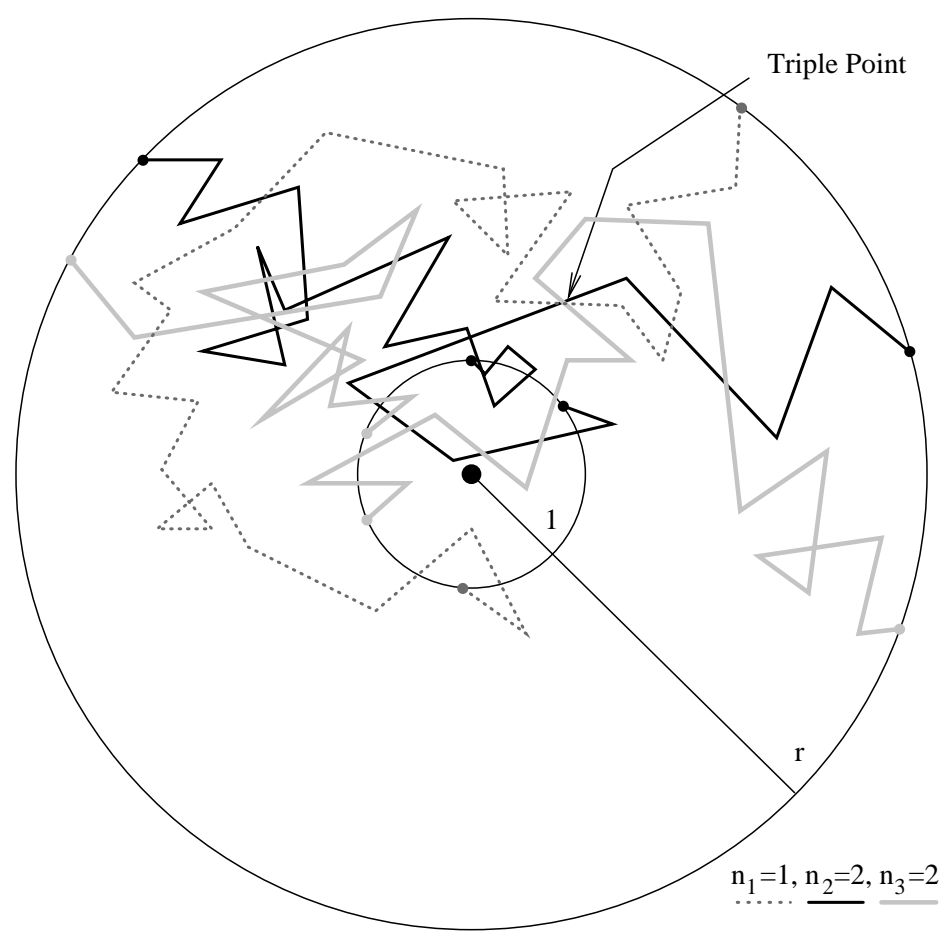

Figure 1: Illustration of a triple point $(p=3)$ with $n_{1}=1, n_{2}=2$ and $n_{3}=2$.

nontrivial exponents

$$
\varsigma_{p}\left(n_{1}, \ldots, n_{p}\right)=-\lim _{t \rightarrow \infty} \frac{2}{\log t} \log \mathbb{P}\left\{\bigcup_{j=1}^{n_{1}} B_{j}^{1}[0, t] \cap \ldots \cap \bigcup_{j=1}^{n_{p}} B_{j}^{p}[0, t]=\emptyset\right\}
$$

exist, see Theorem 1 and the subsequent remark. In Theorem 2 we show that, for $2 \leq n_{3} \leq \cdots \leq n_{p}$, we have

$$
\varsigma_{p}\left(1,2, n_{3}, \ldots, n_{p}\right)=2 .
$$

These are the only exponents we could determine exactly beyond the well-known case of $p=2$. Rigorous proofs of both theorems are given in Section 2.

The bulk of this paper is devoted to the presentation of a detailed numerical study of the values of the, in our opinion, most interesting remaining exponents, see Section 3. One of the motivations of this study was to test the conjecture, motivated by Theorem 2, that the value of the exponents $\varsigma_{p}\left(n_{1}, n_{2}, n_{3}, \ldots, n_{p}\right)$ depend only on the two smallest parameters. This conjecture was not supported by our numerical investigations.

Finally, we remark that we have not been able to use either SLE techniques or quantum gravity to derive even a non-rigorous exact prediction of the exponents if $p>2$. We hope however that our numerical study triggers interest in this problem and that, as in the motivational examples discussed above, future research will address the question of exact formulas for multiple intersection exponents. 


\subsection{Statement of the main theorems}

Let $p \geq 2$ and $n_{1}, \ldots, n_{p}$ be positive integers and $B_{1}^{1}, \ldots, B_{n_{1}}^{1} ; \ldots ; B_{1}^{p}, \ldots, B_{n_{p}}^{p}$ independent planar Brownian motions started uniformly on the unit circle $\partial \mathcal{B}(0,1)$. We define $p$ packets by

$$
\mathfrak{B}^{1}(r):=\bigcup_{j=1}^{n_{1}} B_{j}^{1}\left[0, \tau_{j}^{1}(r)\right], \ldots, \mathfrak{B}^{p}(r):=\bigcup_{j=1}^{n_{p}} B_{j}^{p}\left[0, \tau_{j}^{p}(r)\right],
$$

where $\tau_{j}^{i}(r):=\inf \left\{t \geq 0:\left|B_{j}^{i}(t)\right|=r\right\}$ and $r \geq 1$.

Theorem 1. The limit

$$
\varsigma_{p}\left(n_{1}, \ldots, n_{p}\right):=-\lim _{r \rightarrow \infty} \frac{1}{-\log r} \log \mathbb{P}\left\{\mathfrak{B}^{1}(r) \cap \ldots \cap \mathfrak{B}^{p}(r)=\emptyset\right\}
$$

exists and is positive and finite.

\section{Remarks:}

- Using a standard argument, see [13, Lemma 3.14], one can replace the paths stopped upon hitting the circle of radius $r$, by paths running for $t=r^{2}$ time units. This leads to the characterisation of the exponents given in the overview.

- For $p=2$ all exponents are known, see $[14,15,16]$ :

$$
\varsigma_{2}\left(n_{1}, n_{2}\right)=\frac{\left(\sqrt{24 n_{1}+1}+\sqrt{24 n_{2}+1}-2\right)^{2}-4}{48} .
$$

The technique used to identify the exponents, which is based on the Schramm-Loewner evolution (SLE), does not seem to allow us to identify the exponents for $p>2$.

- We conjecture that one can strengthen this result, as this was done for $p=2$ in [12], and show that there exists a constant $c>0$, depending on the starting points, such that

$$
\lim _{r \rightarrow \infty} r^{\varsigma_{p}\left(n_{1}, \ldots, n_{p}\right)} \mathbb{P}\left\{\mathfrak{B}^{1}(r) \cap \ldots \cap \mathfrak{B}^{p}(r)=\emptyset\right\}=c .
$$

However, this is quite subtle and would go beyond the scope of this paper.

There is a trivial symmetry of the exponents, namely for every permutation $\sigma \in \operatorname{Sym}(p)$, we have

$$
\varsigma_{p}\left(n_{1}, \ldots, n_{p}\right)=\varsigma_{p}\left(n_{\sigma(1)}, \ldots, n_{\sigma(p)}\right) .
$$

Moreover, there are two trivial monotonicity rules for these exponents

(A) $\varsigma_{p}\left(n_{1}, \ldots, n_{p}\right) \leq \varsigma_{p-1}\left(n_{1}, \ldots, n_{p-1}\right)$,

(B) $\varsigma_{p}\left(n_{1}, \ldots, n_{p}\right) \leq \varsigma_{p}\left(m_{1}, \ldots, m_{p}\right)$, if $n_{i} \leq m_{i}$ for $i=1, \ldots, p$. 
As a result of the symmetry of the exponents, we may henceforth assume that the arguments of the exponents are increasing in size, i.e. $n_{1} \leq \cdots \leq n_{p}$. There is one interesting situation in which we can determine the exponents explicitly.

Theorem 2. We have $\varsigma_{p}\left(1,2, n_{3}, \ldots, n_{p}\right)=2$ for any $p \geq 2$ and $2 \leq n_{3} \leq \cdots \leq n_{p}$.

As $\varsigma_{p}(1,2, \ldots, 2) \leq \varsigma_{p}\left(1,2, n_{3}, \ldots, n_{p}\right) \leq \varsigma_{2}(1,2)$ by the monotonicity rules, it suffices to show that

$$
\varsigma_{p}(1,2, \ldots, 2)=2 \text {. }
$$

The proof of this fact is based on the technique of hitting the intersection of $p-1$ Brownian paths by a further path, using an idea of Lawler, see [10] or [11, Section 3], originally used to determine the exponent $\varsigma_{2}(1,2)=2$.

Remark: The definition of the exponents $\varsigma_{p}\left(n_{1}, \ldots, n_{p}\right)$ can be naturally extended to a real argument $\lambda>0$ in place of $n_{p}$ by letting

$$
\begin{aligned}
& \varsigma_{p}\left(n_{1}, \ldots, n_{p-1}, \lambda\right):= \\
& \quad-\lim _{r \rightarrow \infty} \frac{1}{-\log r} \log \mathbb{E}\left[\mathbb{P}\left\{\mathfrak{B}^{1}(r) \cap \ldots \cap \mathfrak{B}^{p-1}(r) \cap B_{1}^{p}\left[0, \tau_{1}^{p}(r)\right]=\emptyset \mid \mathfrak{B}^{1}(r), \ldots, \mathfrak{B}^{p-1}(r)\right\}^{\lambda}\right] .
\end{aligned}
$$

The mapping $\lambda \mapsto \varsigma_{p}\left(n_{1}, \ldots, n_{p-1}, \lambda\right)$ cannot always be analytic: for instance, recall that $\varsigma_{3}(1,2,1) \leq$ $\varsigma_{2}(1,1)=\frac{5}{4}$, but $\varsigma_{3}(1,2, \lambda)=2$ for all $\lambda \geq 2$, by Theorem 2 and the monotonicity rules. However, for $p=2$ this mapping is analytic, see [17].

\subsection{Conjectures}

In this section we formulate the main conjecture motivated by our numerical studies. A detailed description of these studies and their outcomes will be given in Section 3.

Let $p \in \mathbb{N}$ and $n_{1}, \ldots, n_{p} \in \mathbb{N}$ with $n_{1} \leq n_{2} \leq \cdots \leq n_{p}$. Define

$$
k:=\max \left\{2, \min \left\{\ell \in\{1, \ldots, p\}: n_{\ell+1}>n_{\ell}\right\}\right\},
$$

with $k:=p$ if the set is empty. We conjecture that

$$
\varsigma_{p}\left(n_{1}, \ldots, n_{p}\right)=\varsigma_{k}\left(n_{1}, \ldots, n_{k}\right) .
$$

In fact, this holds, by Theorem 2 for the case $k=2, n_{k}=2$, and we have numerical evidence for

- $\varsigma_{3}(1,1,2)=1.2503 \pm 0.0011$ to be compared with $\varsigma_{2}(1,1)=\frac{5}{4}$

- $\varsigma_{4}(1,1,1,2)=1.02 \pm 0.004$ to be compared with $\varsigma_{3}(1,1,1)=1.027 \pm 0.005$

- $\varsigma_{3}(2,2,3)=2.937 \pm 0.01 \quad$ to be compared with $\varsigma_{2}(2,2) \quad=\frac{35}{12}=2.91666 \ldots$

This is evidence that if $p$ packets of Brownian motions are required not to intersect, this is achieved by the $k$ smallest packets not intersecting, if these are strictly smaller than the $p-k$ largest packets. Beyond this conjecture it is interesting to compare further values, namely 
- $\varsigma_{3}(1,3,3)=2.688 \pm 0.01$ with $\varsigma_{2}(1,3)=\frac{13+\sqrt{73}}{8}=2.693000 \ldots$

- $\varsigma_{3}(2,3,3)=3.767 \pm 0.06$ with $\varsigma_{2}(2,3)=\frac{47+5 \sqrt{73}}{24}=3.738334113 \ldots$,

which is evidence supporting the conjecture that in some cases nonintersection is achieved by the two smallest packets not intersecting, even if the second and third smallest have the same size. However this cannot be expected in all situations, as can be seen comparing

- $\varsigma_{3}(1,1,1)=1.027 \pm 0.005$ with $\varsigma_{2}(1,1)=\frac{5}{4}$.

\section{Proofs of Theorems 1 and 2.}

\subsection{Proof of Theorem 1}

Denote by $x=\left(x_{1}^{1}, \ldots, x_{n_{1}}^{1} ; \ldots ; x_{1}^{p}, \ldots, x_{n_{p}}^{p}\right)$ vectors with $n_{1}+\cdots+n_{p}$ entries in $\mathbb{R}^{2}$, playing the role of configurations of our motions at time zero. Consider

$$
a_{r}:=\sup _{\left|x_{j}^{i}\right|=1} \mathbb{P}_{x}\left\{\mathfrak{B}^{1}(r) \cap \cdots \cap \mathfrak{B}^{p}(r)=\emptyset\right\},
$$

where the subindex of $\mathbb{P}$ indicates the starting points of the Brownian motions. Using the strong Markov property and Brownian scaling, we get, for any $r, s \geq 1$,

$$
\begin{aligned}
& a_{r s} \leq \sup _{\left|x_{j}^{i}\right|=1} \mathbb{P}_{x}\left\{\bigcup_{j=1}^{n_{1}} B_{j}^{1}\left[0, \tau_{j}^{1}(r)\right] \cap \cdots \cap \bigcup_{j=1}^{n_{p}} B_{j}^{p}\left[0, \tau_{j}^{p}(r)\right]=\emptyset,\right. \\
& \left.\bigcup_{j=1}^{n_{1}} B_{j}^{1}\left[\tau_{j}^{1}(r), \tau_{j}^{1}(r s)\right] \cap \cdots \cap \bigcup_{j=1}^{n_{p}} B_{j}^{p}\left[\tau_{j}^{p}(r), \tau_{j}^{p}(r s)\right]=\emptyset\right\} \\
& =\sup _{\left|x_{j}^{i}\right|=1} \mathbb{E}_{x}\left[1\left\{\bigcup_{j=1}^{n_{1}} B_{j}^{1}\left[0, \tau_{j}^{1}(r)\right] \cap \cdots \cap \bigcup_{j=1}^{n_{p}} B_{j}^{p}\left[0, \tau_{j}^{p}(r)\right]=\emptyset\right\}\right. \\
& \left.\times \mathbb{P}_{\left(B_{j}^{i}\left(\tau_{j}^{i}(r)\right)\right.}\left\{\bigcup_{j=1}^{n_{1}} B_{j}^{1}\left[\tau_{j}^{1}(r), \tau_{j}^{1}(r s)\right] \cap \cdots \cap \bigcup_{j=1}^{n_{p}} B_{j}^{p}\left[\tau_{j}^{p}(r), \tau_{j}^{p}(r s)\right]=\emptyset\right\}\right] \\
& \leq a_{r} a_{s} .
\end{aligned}
$$

Hence the function given by $b_{t}:=\log a_{2^{t}}$ is subadditive and, by the subadditivity lemma, see e.g. [11, Lemma 5.2.1], we thus have $\lim _{t \rightarrow \infty} b_{t} / t=\inf _{t>0} b_{t} / t$. Therefore,

$$
\tilde{\varsigma}_{p}\left(n_{1}, \ldots, n_{p}\right):=-\lim _{r \rightarrow \infty} \frac{1}{\log r} \log \sup _{\left|x_{j}^{i}\right|=1} \mathbb{P}_{x}\left\{\mathfrak{B}^{1}(r) \cap \cdots \cap \mathfrak{B}^{p}(r)=\emptyset\right\}
$$

exists, and is positive.

Next, we show that we can replace the optimised starting points by starting points uniformly chosen from the unit circle. Clearly, we have

$$
\mathbb{P}\left\{\mathfrak{B}^{1}(r) \cap \cdots \cap \mathfrak{B}^{p}(r)=\emptyset\right\} \leq \sup _{\left|x_{j}^{i}\right|=1} \mathbb{P}_{x}\left\{\mathfrak{B}^{1}(r) \cap \cdots \cap \mathfrak{B}^{p}(r)=\emptyset\right\},
$$


where $\mathbb{P}$ refers to the original scenario of Brownian motions started uniformly on the unit circle.

Conversely, using the Markov property, for $r>2$, we have

$$
\begin{aligned}
& \sup _{\left|x_{j}^{i}\right|=1} \mathbb{P}_{x}\left\{\mathfrak{B}^{1}(r) \cap \cdots \cap \mathfrak{B}^{p}(r)=\emptyset\right\} \\
& \quad \leq \sup _{\left|x_{j}^{i}\right|=1} \mathbb{E}_{x}\left[\mathbb{P}_{\left(B_{j}^{i}\left(\tau_{j}^{i}(2)\right)\right)}\left\{\bigcup_{j=1}^{n_{1}} B_{j}^{1}\left[\tau_{j}^{1}(2), \tau_{j}^{1}(r)\right] \cap \cdots \cap \bigcup_{j=1}^{n_{p}} B_{j}^{p}\left[\tau_{j}^{p}(2), \tau_{j}^{p}(r)\right]=\emptyset\right\}\right] .
\end{aligned}
$$

By the Harnack principle, the law of the vector $\left(B_{j}^{i}\left(\tau_{j}^{i}(2)\right)\right)$ is bounded, uniformly in $x$, by a constant multiple of the uniform distribution on the $\left(n_{1}+\cdots+n_{p}\right)$-fold cartesian power of the circle $\partial \mathcal{B}(0,2)$. Denoting this constant by $C$ and using Brownian scaling,

$$
\begin{aligned}
& \mathbb{P}\left\{\bigcup_{j=1}^{n_{1}} B_{j}^{1}\left[0, \tau_{j}^{1}(r / 2)\right]\right.\left.\cap \cdots \cap \bigcup_{j=1}^{n_{p}} B_{j}^{p}\left[0, \tau_{j}^{p}(r / 2)\right]=\emptyset\right\} \\
& \geq C^{-1} \sup _{\left|x_{j}^{i}\right|=1} \mathbb{P}_{x}\left\{\mathfrak{B}^{1}(r) \cap \cdots \cap \mathfrak{B}^{p}(r)=\emptyset\right\} .
\end{aligned}
$$

Combining (2.1) and (2.2) yields that

$$
\varsigma_{p}\left(n_{1}, \ldots, n_{p}\right):=-\lim _{r \rightarrow \infty} \frac{1}{\log r} \log \mathbb{P}\left\{\mathfrak{B}^{1}(r) \cap \cdots \cap \mathfrak{B}^{p}(r)=\emptyset\right\}
$$

exists and coincides with $\tilde{\varsigma}_{p}\left(n_{1}, \ldots, n_{p}\right)$. Note, finally, that the monotonicity rule (A) implies that $\varsigma_{p}\left(n_{1}, \ldots, n_{p}\right) \leq \varsigma_{2}\left(n_{1}, n_{2}\right)<\infty$, and hence the exponents are positive and finite.

\subsection{Proof of Theorem 2}

Recall that it suffices to show (1.1). We start by formulating the key lemma. We let $W^{1}, \ldots, W^{p}$ be independent Brownian paths. For $r, s>0$ denote by $\tau^{i}(x, r)$ the first hitting time by the motion $W^{i}$ of the circle $\partial \mathcal{B}(x, r)$ with centre $x$ and radius $r$, and let $\tau^{i}(x, r, s)$ be the first hitting time of $\partial \mathcal{B}(x, s)$ after $\tau^{i}(x, r)$.

Lemma 3. Fix $x \in \mathcal{B}(0,1)$. Suppose that $W^{1}, \ldots, W^{p}$ are independent Brownian paths started uniformly on the circle $\partial \mathcal{B}(0,2)$. Define the set

$$
\mathfrak{W}:=\bigcap_{j=2}^{p} W^{j}\left[0, \tau^{j}(0,4)\right]
$$

and the events

$$
\begin{aligned}
& E_{x, r}=\left\{W^{1}\left[0, \tau^{1}(x, r / 2)\right] \cap \mathfrak{W}=\emptyset\right\} \\
& N_{x, r}=\left\{W^{1}\left[0, \tau^{1}(x, r / 2, r)\right] \cap \mathfrak{W} \neq \emptyset\right\} \\
& H_{x, r}=\left\{\tau^{i}(x, r / 2)<\tau^{i}(0,4) \text { for all } i=1, \ldots, p\right\}
\end{aligned}
$$

Then

$$
\liminf _{r \downarrow 0} \frac{1}{|\log r|} \log \mathbb{P}\left[E_{x, r} \cap N_{x, r} \mid H_{x, r}\right] \geq-\varsigma_{p}(1,2, \ldots, 2) .
$$


Let us first see how (1.1) follows from this lemma. Let

$$
\tau=\inf \left\{t>0: W^{1}(t) \in \mathfrak{W}\right\} .
$$

Now let $\mathfrak{B}$ be a collection of pairwise disjoint discs of fixed radius $0<r<1 / 2$ with centres in the disc $\mathcal{B}(0,1)$, which has cardinality at least $(2 r)^{-2}$. Then, obviously,

$$
1 \geq \mathbb{P}\left\{W^{1}\left[0, \tau^{1}(0,4)\right] \cap \mathfrak{W} \neq \emptyset\right\} \geq \sum_{\mathcal{B} \in \mathfrak{B}} \mathbb{P}\left\{W^{1}(\tau) \in \mathcal{B}, \tau<\tau^{1}(0,4)\right\} .
$$

Now, fix a disc $\mathcal{B}=\mathcal{B}(x, r) \in \mathfrak{B}$. The event $\left\{W^{1}(\tau) \in \mathcal{B}, \tau<\tau^{1}(0,4)\right\}$ is implied by the events

$$
E_{x, r} \cap N_{x, r} \cap\left\{\tau^{1}(x, r / 2)<\tau^{1}(0,4)\right\} .
$$

Recall that

$$
\mathbb{P}\left[H_{x, r}\right]=\mathbb{P}\left\{\tau^{1}(x, r / 2)<\tau^{1}(0,4)\right\}^{p}=r^{o(1)} .
$$

Combining this with Lemma 3 , for any $\varepsilon>0$ and sufficiently small $r>0$,

$$
\mathbb{P}\left\{W^{1}(\tau) \in \mathcal{B}, \tau<\tau^{1}(0,4)\right\} \geq r^{\varsigma_{p}(1,2, \ldots, 2)+\varepsilon} .
$$

This implies

$$
1 \geq \sum_{\mathcal{B} \in \mathfrak{B}} r^{\varsigma_{p}(1,2, \ldots, 2)+\varepsilon} \geq r^{-2+\varsigma_{p}(1,2, \ldots, 2)+2 \varepsilon},
$$

and therefore $\varsigma_{p}(1,2, \ldots, 2) \geq 2-2 \varepsilon$. The lower bound follows as $\varepsilon>0$ was arbitrary, and the upper bound in (1.1) follows from $\varsigma_{p}(1,2, \ldots, 2) \leq \varsigma_{2}(1,2)=2$, as is known from $[10,11]$.

Proof of Lemma 3. Before we describe the technical details we sketch the idea of the proof. Since the paths of $p$ planar Brownian motions intersect with positive probability, by Brownian scaling, the conditional probability of $N_{x, r}$ given $H_{x, r}$ is bounded from below as $r \rightarrow 0$. Hence this condition can be neglected when computing the probability in Lemma 3 . For $j=1, \ldots, p$ we decompose the paths $W^{j}$ into the pieces $W^{j}\left[0, \tau^{j}(x, r / 2)\right]$ and $W^{j}\left[\tau^{j}(x, r / 2), \tau^{j}(0,4)\right]$. By time reversal for $W^{j}\left[0, \tau^{j}(x, r / 2)\right]$, we can compare the probability in question with the non-intersection probability for packets of size $n_{1}=1, n_{2}=\cdots=n_{p}=2$, which is of order $\approx r^{\varsigma_{p}(1,2, \ldots, 2)}$.

We now come to the technical details, see the appendix in [22] for the necessary facts about Brownian excursions between concentric spheres. Let $\varrho^{1}=r$ and $\varrho^{j}=r / 2$ for $j=2, \ldots, p$. Conditioned on $\left\{\tau^{i}\left(x, \varrho^{j} / 2\right)<\tau^{i}(x, 3)\right\}$ the path $W^{i}\left[0, \tau^{i}\left(x, \varrho^{j} / 2\right)\right]$ is contained in an excursion from $\partial \mathcal{B}(x, 3)$ to $\partial \mathcal{B}\left(x, \varrho^{j} / 2\right)$. The time-reversal of this excursion is contained in the path of a Brownian motion $\widetilde{W}^{i}$ started uniformly on $\partial \mathcal{B}\left(x, \varrho^{j} / 2\right)$ and stopped upon reaching $\partial \mathcal{B}(x, 3)$, say at time $\widetilde{\tau}^{i}(x, 3)$. Analogously to $(2.3)$ and (2.4) define the set

$$
\widetilde{\mathfrak{W}}=\bigcap_{j=2}^{p}\left(\widetilde{W}^{j}\left[0, \widetilde{\tau}^{j}(x, 3)\right] \cup W^{j}\left[\tau^{j}(x, r / 4, r / 2), \tau^{j}(0,4)\right]\right),
$$

and the events

$$
\begin{aligned}
\widetilde{E}_{x, r} & =\left\{\widetilde{W}^{1}\left[0, \widetilde{\tau}^{1}(x, 3)\right] \cap \widetilde{\mathfrak{W}}=\emptyset\right\}, \\
\widetilde{N}_{x, r} & =\left\{\bigcap_{j=1}^{p} W^{j}\left[\tau^{j}\left(x, \rho^{j} / 2\right), \tau^{j}\left(x, \rho^{j} / 2, \rho^{j}\right)\right] \neq \emptyset\right\}, \\
\widetilde{H}_{x, r} & =\left\{\tau^{j}\left(x, \varrho^{j} / 2\right)<\tau^{j}(x, 3) \text { for all } j=1, \ldots, p\right\} .
\end{aligned}
$$


Note that $W^{1}\left[0, \tau^{1}\left(x, \rho^{1}\right)\right] \cap \mathcal{B}(x, r / 2)=\emptyset$ and $W^{j}\left[\tau^{j}\left(x, \rho^{j} / 2\right), \tau^{j}\left(x, \rho^{j} / 2, \rho^{j}\right)\right) \subset \mathcal{B}(x, r / 2)$ for $j=$ $2, \ldots, p$. Hence

$$
W^{1}\left[0, \tau^{1}\left(x, \rho^{1}\right)\right] \cap(\mathfrak{W} \backslash \widetilde{\mathfrak{W}}) \subset W^{1}\left[0, \tau^{1}\left(x, \rho^{1}\right)\right] \cap \bigcap_{j=2}^{p} W^{j}\left[\tau^{j}\left(x, \rho^{j} / 2\right), \tau^{j}\left(x, \rho^{j} / 2, \rho^{j}\right)\right)=\emptyset
$$

which implies $\widetilde{E}_{x, r} \subset E_{x, r}$. Note that trivially, we have $\widetilde{H}_{x, r} \subset H_{x, r}$ and $\widetilde{N}_{x, r} \subset N_{x, r}$ which implies

$$
E_{x, r} \cap N_{x, r} \cap H_{x, r} \supset \widetilde{E}_{x, r} \cap \widetilde{N}_{x, r} \cap \widetilde{H}_{x, r}
$$

Finally, note that

$$
f(x, r):=\frac{\mathbb{P}\left[\widetilde{H}_{x, r}\right]}{\mathbb{P}\left[H_{x, r}\right]}=\frac{\mathbb{P}\left\{\tau^{1}\left(x, \varrho^{1} / 2\right)<\tau^{1}(x, 3)\right\}^{p}}{\mathbb{P}\left\{\tau^{1}(x, r / 2)<\tau^{1}(0,4)\right\}^{p}} \geq \frac{1}{2}
$$

for all $x$ and for sufficiently small values of $r>0$.

By (2.5), (2.6) and the definition of the conditional probability, we conclude

$$
\mathbb{P}\left[E_{x, r} \cap N_{x, r} \mid H_{x, r}\right] \geq f(x, r) \mathbb{P}\left[\widetilde{E}_{x, r} \cap \widetilde{N}_{x, r} \mid \widetilde{H}_{x, r}\right] .
$$

Fix $\varepsilon>0$. Invoking the definition of the exponent, the Harnack principle and Brownian scaling, for sufficiently small $r>0$,

$$
\mathbb{P}\left[\widetilde{E}_{x, r} \mid \widetilde{H}_{x, r}\right] \geq r^{\varsigma_{p}(1,2, \ldots, 2)+\varepsilon} .
$$

Define the compact sets

$$
\begin{aligned}
C & :=\left\{y=\left(y^{1}, \ldots, y^{p}\right): y^{j} \in \partial \mathcal{B}\left(0, \varrho^{j} / 2\right) \text { for } j=1, \ldots, p\right\} \text { and } \\
D & :=\left\{z=\left(z^{1}, \ldots, z^{p}\right): z^{j} \in \partial \mathcal{B}\left(0, \varrho^{j}\right) \text { for } j=1, \ldots, p\right\} .
\end{aligned}
$$

For $y \in C$ and $z \in D$ let $\left(\bar{W}^{j}, j=1, \ldots, p\right)$ be an independent family of Brownian motions where each motion $\bar{W}^{j}$ is started at $y^{j}$ and is conditioned to leave $\mathcal{B}\left(0, \varrho^{j}\right)$ at $z^{j}$ (at time $\bar{\tau}^{j}$ ). Denote by $\mathbb{P}_{y, z}$ the corresponding probability measure. It is easy to see that the map

$$
\phi: C \times D \rightarrow[0,1], \quad(y, z) \mapsto \mathbb{P}_{y, z}\left\{\bar{W}^{1}\left[0, \tau^{1}\right] \cap \ldots \cap \bar{W}^{p}\left[0, \tau^{p}\right] \neq \emptyset\right\}
$$

is continuous and strictly positive, and independent of $r$ by Brownian scaling. Hence

$$
c:=\inf _{y \in C, z \in D} \phi(y, z)>0 .
$$

We infer that

$$
\mathbb{P}\left[\widetilde{N}_{x, r} \mid \widetilde{E}_{x, r} \cap \widetilde{H}_{x, r}\right] \geq c>0 .
$$

Hence, combing our results, for sufficiently small $r>0$

$$
\mathbb{P}\left[\widetilde{E}_{x, r} \cap \widetilde{N}_{x, r} \mid \widetilde{H}_{x, r}\right]=\mathbb{P}\left[\widetilde{E}_{x, r} \mid \widetilde{H}_{x, r}\right] \mathbb{P}\left[\widetilde{N}_{x, r} \mid \widetilde{E}_{x, r} \cap \widetilde{H}_{x, r}\right] \geq c r^{\varsigma_{p}(1,2, \ldots, 2)+\varepsilon},
$$

and this completes the proof as $\varepsilon>0$ was arbitrary. 


\section{Simulations}

To get hold of those exponents which we could not determine explicitly, we have performed Monte Carlo simulations. This has successfully generated conjectures in the $p=2$ case, see Duplantier and Kwon [6], Li and Sokal [20] and Burdzy, Lawler and Polaski [1].

3.1 The general scheme. Before we list and analyse the simulated data, we explain how we got it. Fix positive integers $p$ and $n_{1}, \ldots, n_{p}$. The aim is to get an estimate on $\varsigma_{p}\left(n_{1}, \ldots, n_{p}\right)$. Instead of Brownian motions we simulate two-dimensional symmetric nearest neighbour random walks. As it reduces computing effort, we work with boxes rather than with discs. (For comparison we have performed some of the simulations also with discs and there was no significant difference in the results.) First we fix an increasing sequence of box half-lengths $L_{0}, \ldots, L_{K}$ (in most cases $L_{k+1}=\left\lfloor 1.1 \cdot L_{k}\right\rfloor$ and the maximal value $m=L_{L}$ restricted to 20000, 40000 or 80000) and the sample size $N$ of the simulation.

Step 1. We start $n_{1}+\ldots+n_{p}$ independent random walks at the origin $0 \in \mathbb{Z}^{2}$ and stop each of them when it hits the (graph) boundary of the box $\left\{-L_{0}, \ldots, L_{0}\right\}^{2}=\left[-L_{0}, L_{0}\right]^{2} \cap \mathbb{Z}^{2}$. This defines the starting positions of the random walks.

Step 2. Assume we are at level $k$ (after Step 1 we are at level $k=1$ ). Independently run the random walks until they hit the boundary of the box $\left\{-L_{k}, \ldots, L_{k}\right\}^{2} \subset \mathbb{Z}^{2}$. Separately, keep track of the set $A_{k, i} \subset\left\{-L_{k}+1, \ldots, L_{k}-1\right\}^{2}$ of points that are visited by the $i$ th package of $n_{i}$ random walks before hitting the boundary of $\left\{-L_{k}, \ldots, L_{k}\right\}^{2}$ (after Step 1).

If $A_{k, 1} \cap \ldots \cap A_{k, p}=\emptyset$, then we say that we have survived level $k$ and we enter level $k+1$ (that is, we perform Step 2 again with $k$ replaced by $k+1)$. Otherwise we stop this sample and start a new simulation in Step 1.

By $N_{k}$ we denote the number of samples that have survived level $k$. Clearly, $N_{0}=N$. We should have

$$
N_{k} / N \approx\left(L_{k} / L_{0}\right)^{-\varsigma_{p}\left(n_{1}, \ldots, n_{p}\right)} .
$$

Hence in a double logarithmic plot of $\log \left(N_{k}\right)$ against $\log \left(L_{k}\right)$ the points should be on a line with slope $-\varsigma_{p}\left(n_{1}, \ldots, n_{p}\right)$. Linear regression then gives an estimate for the exponent $\varsigma_{p}\left(n_{1}, \ldots, n_{p}\right)$.

As it turns out that a line can be fitted well only for large values of $L_{k}$, we have neglected the small values of $L_{k}$ in order to get a reasonable estimate for $\varsigma_{p}\left(n_{1}, \ldots, n_{p}\right)$. In Figure 3.4.1 below we plotted the data points used for the linear regression with solid circles, the other points with hollow circles.

As can be seen from Figure 3.4.1, for $\xi(1,1)$ this gives a pretty good estimate of the exact value $\frac{5}{4}$, even with a moderate computing effort of about 2000 hours CPU time. However, for $\xi(1,1,1)$ the points tend to lie on a straight line only for large values of $L_{k}$ and thus require

(i) a large maximal box size $m=L_{K}$ and thus a big computer memory of size $(2 m+1)^{2}$ bytes in order to keep track of the visited points,

(ii) a large sample size $N_{0}$ in order that $N_{K} \approx N_{0} \cdot\left(L_{K} / L_{0}\right)^{-\xi(1,1,1)}$ is big enough to obtain reliable data from the simulation.

Since the CPU time we need for each sample grows with $m$, (i) and (ii) imply that we need huge amounts of CPU time. Furthermore, with huge sample sizes and box sizes, we run into the order of the cycle length of the common 48 bit linear congruence random number generators. 
The computations were performed on different computers, mainly on two parallel Linux clusters at the University of Mainz on Opteron 2218 processors with $2.6 \mathrm{GHz}$ and on Opteron 244 processors with $1.8 \mathrm{GHz}$. The programme code is written in C. As random number generator we used drand64(), a 64 bit linear congruence generator following the rule

$$
r_{n+1}=\left(a r_{n}+c\right) \bmod 2^{64}
$$

with

$$
a=6364136223846793005 \text { and } c=1
$$

(see [9, pp106-108]).

The linear regression method does not give a quantitative estimate on the statistical error. In order to get such an error estimate we did the following. Having in mind that the systematic error is large for small box sizes, we choose a minimal box number $k_{\min } \in\{1, \ldots, K-1\}$ and neglect the data from all smaller boxes. Furthermore, we pretend that the asymptotics for $p_{L}$ is exact for $k \geq k_{\min }$, that is,

$$
p_{L_{k}}=C L_{k}^{-\varsigma} \text { for all } k \geq k_{\min }
$$

for some $C>0$. In particular, the conditional probability to have no multiple intersections before leaving $B_{L_{k+1}}$ given there is no multiple intersection before leaving $B_{L_{k}}$ is

$$
\bar{p}_{k}:=\frac{p_{L_{k+1}}}{p_{L_{k}}}=\left(\frac{L_{k}}{L_{k+1}}\right)^{-\varsigma}=: q_{k}^{-\varsigma}
$$

Here the likelihood function for the observation

$$
\left(N_{k_{\min }}, N_{k_{\min }+1}, \ldots, N_{K}\right)=n:=\left(n_{k_{\min }}, n_{k_{\min }+1}, \ldots, n_{K}\right)
$$

is

$$
\begin{aligned}
L_{n}(\varsigma) & =C(n) \prod_{l=k_{\min }}^{K-1} \bar{p}_{l}^{n_{l+1}}\left(1-\bar{p}_{l}\right)^{n_{l}-n_{l+1}} \\
& =C(n) \prod_{l=k_{\min }}^{K-1} \bar{q}_{l}^{\varsigma_{l+1}}\left(1-\bar{q}_{l}^{\varsigma}\right)^{n_{l}-n_{l+1}}
\end{aligned}
$$

for some $C(n)>0$. The log-likelihood function is

$$
\mathcal{L}_{n}(\varsigma)=\log C(n)+\sum_{l=k_{\min }}^{K-1}\left(n_{l+1} \varsigma \log \left(q_{l}\right)+\left(n_{l}-n_{l+1}\right) \log \left(1-q_{l}^{\varsigma}\right)\right) .
$$

The maximum likelihood estimator (MLE) $\hat{\varsigma}$ is defined by

$$
\mathcal{L}_{n}(\hat{\varsigma})=\sup _{\varsigma>0} \mathcal{L}_{n}(\varsigma) .
$$

We compute the derivatives

$$
\mathcal{L}_{n}^{\prime}(\varsigma)=\sum_{l=k_{\min }}^{K-1} n_{l+1} \log \left(q_{l}\right)-\sum_{l=k_{\min }}^{K-1}\left(n_{l}-n_{l+1}\right) \frac{\log \left(q_{l}\right) q_{l}^{\varsigma}}{1-q_{l}^{\varsigma}}
$$


and

$$
\mathcal{L}_{n}^{\prime \prime}(\varsigma)=-\sum_{l=k_{\min }}^{K-1}\left(n_{l}-n_{l+1}\right) \frac{\left(\log \left(q_{l}\right)\right)^{2} q_{l}^{\varsigma}}{\left(1-q_{l}^{\varsigma}\right)^{2}} .
$$

Clearly, $\mathcal{L}_{n}^{\prime \prime}(\varsigma)<0$, hence $\varsigma \mapsto \mathcal{L}_{n}(\varsigma)$ is strictly concave and thus $\hat{\varsigma}$ is the unique solution of

$$
\mathcal{L}_{n}^{\prime}(\hat{\varsigma})=0 .
$$

Hence, for given data, the MLE can easily be computed numerically (we used a Newton approximation scheme).

Denote by $\hat{\varsigma}_{n_{0}}$ the MLE for sample size $n_{0}$. By standard theory for MLEs, $(\hat{\varsigma})_{n_{0} \in \mathbb{N}}$ is consistent and asymptotically normally distributed. In fact, by Corollary 6.2 .1 of [19],

$$
\hat{\varsigma}_{n_{0}} \stackrel{n_{0} \rightarrow \infty}{\longrightarrow} \varsigma \text { stochastically. }
$$

Furthermore, by [19, Corollary 6.2.3], $(\hat{\varsigma})_{n_{0}}$ is asymptotically efficient (that is, optimal) and by [19, Theorem 6.2.3] (with $\mathcal{N}_{0,1}$ the standard normal distribution)

$$
\sqrt{n_{0} I(\varsigma)}\left(\hat{\varsigma}_{n_{0}}-\varsigma\right) \stackrel{n_{0} \rightarrow \infty}{\longrightarrow} \mathcal{N}_{0,1} \quad \text { in distribution. }
$$

Here

$$
I(\varsigma)=-\mathbb{E}\left[\mathcal{L}_{N}^{\prime \prime}(\varsigma) \mid N_{0}=1\right]=p_{L_{k_{\min }}} \sum_{l=k_{\min }}^{K-1}\left(\prod_{m=k_{\min }}^{l-1} \bar{p}_{m}\right)\left(1-\bar{p}_{l}\right) \frac{\left(\log \left(q_{l}\right)\right)^{2} q_{l}^{\varsigma}}{\left(1-q_{l}^{\varsigma}\right)^{2}}
$$

is the Fisher information for one sample. As we do not know the true value of $\varsigma$ and since we do not know $p_{L_{k_{\min }}}$, we replace $I(\varsigma)$ by

$$
I_{n}(\varsigma)=-\frac{1}{n_{0}} \mathcal{L}_{n}^{\prime \prime}(\varsigma)
$$

By the law of large numbers $I_{N}(\varsigma) \stackrel{n_{0} \rightarrow \infty}{\longrightarrow} I(\varsigma)$ almost surely, uniformly in $\varsigma$ in compact sets. Hence by (3.8), we have $I_{N}(\hat{\varsigma}) \stackrel{n_{0} \rightarrow \infty}{\longrightarrow} I(\varsigma)$ stochastically. Hence we use

$$
\widehat{\sigma}^{2}:=-1 / \mathcal{L}_{N}^{\prime \prime}(\hat{\varsigma})
$$

as an estimator for the variance of $\hat{\varsigma}$ and obtain

$$
\frac{\hat{\varsigma}-\varsigma}{\widehat{\sigma}} \stackrel{n_{0} \rightarrow \infty}{\longrightarrow} \mathcal{N}_{0,1} \quad \text { in distribution. }
$$

Concluding, an asymptotic $95 \%$ confidence interval for $\varsigma$ is given by

$$
[\hat{\varsigma}-2 \widehat{\sigma}, \hat{\varsigma}+2 \widehat{\sigma}] \text {. }
$$

We have performed the simulations for the exponents $\varsigma_{2}(1,1)$ and $\varsigma_{2}(2,2)$ as benchmark problems, and then did the simulations on a larger scale for

$$
\varsigma_{3}(1,1,1), \quad \varsigma_{3}(1,1,2), \quad \varsigma_{4}(1,1,1,1), \quad \varsigma_{4}(1,1,1,2) .
$$


3.2 Two-level scheme. The simulations turn out to be very time-consuming, especially for the exponents with a larger numerical value. In order to get a more efficient scheme in this situation consider the following simplification of the simulation scheme presented above:

Assume there are only three box sizes, $L_{0}$ (about 30), $L_{1}$ (about 10000 ) and $L_{2}=2 L_{1}$. Then (3.7) can be solved explicitly and the maximum likelihood estimator for $\varsigma$ is

$$
\hat{\varsigma}=-\frac{\log \left(n_{2} / n_{1}\right)}{\log (2)}
$$

In order to reduce the variance of $\hat{\varsigma}$ we have to increase $N_{1}$, that is the sample size $n_{0}$. However, since it takes much CPU time to obtain a sample that contributes to $N_{1}$, we may wish to use this very sample as the starting point for a number $m$ of trials running from box size $L_{1}$ to $L_{2}$. Assume that $x$ among these $m$ trials have survived until $L_{2}$ (that is, have reached the boundary of the $L_{2}$-box without producing a multiple intersection), then $p_{S}=\frac{x}{m}$ is an estimator for the conditional probability of producing no multiple intersection until leaving the $L_{2}$-box for the given realisation $S$ of the paths of all walks in the $L_{1}$-box. Now we can prescribe the number $n=n_{1}$ of "master samples" and for $i=1, \ldots, n$ let $x_{i}$ be the corresponding number of surviving trials and write $\widehat{p_{i}}:=x_{i} / m$. Hence for

$$
p:=\frac{p_{L_{2}}}{p_{L_{1}}}=\mathbb{E}\left[p_{S}\right]
$$

we get the unbiased estimator

$$
\hat{p}=\frac{1}{n} \sum_{l=1}^{n} \widehat{p}_{i} .
$$

The unbiased estimator for the variance of $\hat{p}$ is

$$
\widehat{\sigma_{p}^{2}}=\frac{1}{n(n-1)} \sum_{l=1}^{n}\left(\widehat{p}_{i}-\hat{p}\right)^{2} .
$$

From $\hat{p}$ and $\widehat{\sigma_{p}^{2}}$ we obtain the estimators for $\varsigma$ and the variance $\sigma^{2}$ of $\hat{\varsigma}$

$$
\hat{\varsigma}=-\frac{\log (p)}{\log (2)} \quad \text { and } \quad \widehat{\sigma^{2}}=\frac{\widehat{\sigma_{p}^{2}}}{(\log (2) \hat{p})^{2}}
$$

We have employed this scheme for the exponents with numerical values larger than 2 , and we explain now why it is more efficient in these cases.

The expected time planar random walk needs to go from the boundary of $\{-L, \ldots, L\}^{2}$ to the boundary of $\{-L-1, \ldots, L+1\}^{2}$ is of order $L$. The probability that a given sample ever reaches the boundary of $\{-L-1, \ldots, L+1\}^{2}$ is of order $L^{-\varsigma}$. Hence (if we stop the simulation as soon as the first multiple intersection is detected) the expected CPU time for each sample until box size $L_{1}$ is of order

$$
\sum_{L=L_{0}}^{L_{1}} L^{1-\varsigma}
$$

For $\varsigma>2$ this sum is of order 1 , for $\varsigma \leq 2$, it is of order $L_{1}^{2-\varsigma}$. Now the probability that a sample reaches box size $L_{1}$ without producing a multiple intersection is of order $L_{1}^{-\varsigma}$. Hence the expected 
CPU time needed for simulating a "master sample" is of order $L^{2 \vee \varsigma}$. On the other hand, each of the trials started from the master sample needs an expected CPU time of order $L_{1}^{2}$. Hence for $\varsigma>2$ we can run $m=L_{1}^{\varsigma-2}$ trials without increasing the CPU significantly.

In order to make a good choice for $m$, compute the variance of $\hat{p}$

$$
\operatorname{Var}[\hat{p}]=n^{-1} \operatorname{Var}\left[p_{S}\right]+\frac{1}{m n} \mathbb{E}\left[p_{S}\left(1-p_{S}\right)\right] \leq n^{-1} \operatorname{Var}\left[p_{S}\right]+\frac{1}{m n} \mathbb{E}\left[p_{S}\right] .
$$

The quantities $\operatorname{Var}\left[p_{S}\right]$ and $\mathbb{E}\left[p_{S}\right] \approx 2^{-\varsigma}$ can be estimated from a test simulation as well as the expected CPU time $T_{1}$ to produce a master sample and the expected time $T_{2}$ used for each subsequent trial. Now it is an optimisation problem for the total CPU time $n\left(T_{1}+m T_{2}\right)$ versus the variance $\operatorname{Var}[\hat{p}]$. For some of the simulations we have done test runs and solved the optimisation problem. Here $m=1000$ turned out to be a reasonable choice that we have then used in all simulations.

We have performed the simulations according to this scheme with $L_{0}=30, L_{1}=10000, L_{2}=20000$ and $m=1000$ for the exponents

$$
\varsigma_{3}(1,3,3), \quad \varsigma_{3}(2,2,2), \quad \varsigma_{3}(2,2,3), \quad \varsigma_{3}(2,3,3), \quad \varsigma_{4}(2,2,2,2) .
$$

3.3 Numerical results. We present our estimated values $\hat{\varsigma}$ together with a statistical error of $2 \sigma$. For the systematic error it is hard to make a good judgement. From the graphical representation of the results (see below) it seems that for $\varsigma_{3}(1,1,2)$ the systematic error is of a smaller order than the statistical error. For $\varsigma_{3}(1,1,2)$ and $\varsigma_{3}(1,1,1)$ it is presumably of the same order. Finally, for $\varsigma_{4}(1,1,1,1)$ and, even worse for $\varsigma_{5}(1,1,1,1,1)$ we seem to systematically underestimate the values. It would require a lot larger $L_{\max }$ to get more accurate results. For that reason we have not taken too much effort to reduce the statistical error. However, we give the results of the simulations just to provide an idea of the possible values.

\begin{tabular}{|r|l|l|l|r|l|r|r|}
\hline exponent & $\hat{\varsigma}$ & $2 \widehat{\sigma}$ & rigorous & $L_{\min }$ & $L_{\max }$ & $n_{0} / 10^{6}$ & $\begin{array}{c}\mathrm{CPU} \\
\mathrm{time} / \mathrm{h}\end{array}$ \\
\hline$\varsigma_{2}(1,1)$ & 1.2502 & 0.001 & $5 / 4$ & 1069 & 20000 & 500 & 2064 \\
$\varsigma_{2}(2,2)$ & 2.9188 & 0.0033 & $\frac{35}{12}=2.9167$ & 163 & 20000 & 40000 & 1879 \\
$\varsigma_{3}(1,1,1)$ & 1.027 & 0.005 & {$[1 / 2,5 / 4]$} & 18575 & 80000 & 60 & 8262 \\
$\varsigma_{3}(1,1,2)$ & 1.2503 & 0.0011 & {$[1,5 / 4]$} & 1069 & 80000 & 200 & 5858 \\
$\varsigma_{4}(1,1,1,1)$ & 0.877 & 0.006 & {$[1 / 4,5 / 4]$} & 39813 & 80000 & 20 & 18262 \\
$\varsigma_{4}(1,1,1,2)$ & 1.02 & 0.004 & {$[1 / 2,5 / 4]$} & 27194 & 40000 & 200 & 35212 \\
$\varsigma_{5}(1,1,1,1,1)$ & 0.74 & 0.02 & {$[1 / 8,5 / 4]$} & 27194 & 40000 & 0.74 & 1147 \\
\hline
\end{tabular}

Table 1: Numerical results obtained from the first simulation scheme. 


\begin{tabular}{|r|c|l|l|r|r|}
\hline exponent & $\hat{\varsigma}$ & $2 \widehat{\sigma}$ & rigorous & $n$ & $\begin{array}{c}\mathrm{CPU} \\
\text { time } / \mathrm{h}\end{array}$ \\
\hline$\varsigma_{3}(1,3,3)$ & 2.688 & 0.01 & {$[2,(13+\sqrt{73}) / 8]$} & 18100 & 61860 \\
$\varsigma_{3}(2,2,2)$ & 2.786 & 0.01 & {$[2,35 / 12]$} & 16000 & 47943 \\
$\varsigma_{3}(2,2,3)$ & 2.937 & 0.01 & {$[2,35 / 12]$} & 23000 & 116888 \\
$\varsigma_{3}(2,3,3)$ & 3.767 & 0.057 & {$[2,35 / 12]$} & 1000 & 179543 \\
$\varsigma_{4}(2,2,2,2)$ & 2.664 & 0.01 & {$[2,35 / 12]$} & 16000 & 63496 \\
\hline
\end{tabular}

Table 2: Numerical results obtained from the second simulation scheme.

\subsection{Detailed Data.}

\subsubsection{Exponent $\varsigma_{2}(1,1)$.}

The exact value $\varsigma_{2}(1,1)=5 / 4$ is known. This simulation is used as a benchmark test for our simulation.

\begin{tabular}{|r|r|}
\hline$L_{k}$ & $n_{k}$ \\
\hline 30 & 500000000 \\
33 & 455164209 \\
36 & 414185142 \\
39 & 379373384 \\
42 & 349383901 \\
46 & 315390855 \\
50 & 286840826 \\
55 & 257075021 \\
60 & 232385705 \\
66 & 207870728 \\
72 & 187620511 \\
79 & 168084821 \\
86 & 151902122 \\
94 & 136553134 \\
103 & 122326905 \\
\hline
\end{tabular}

\begin{tabular}{|r|r|}
\hline$L_{k}$ & $n_{k}$ \\
\hline 113 & 109366745 \\
124 & 97714439 \\
136 & 87320799 \\
149 & 78109962 \\
163 & 69978568 \\
179 & 62384176 \\
196 & 55800459 \\
215 & 49786852 \\
236 & 44382636 \\
259 & 39563995 \\
284 & 35298660 \\
312 & 31418279 \\
343 & 27932867 \\
377 & 24837149 \\
414 & 22109889 \\
\hline
\end{tabular} \mid \begin{tabular}{rr|r|}
$L_{k}$ & $n_{k}$ \\
505 & 19660552 \\
550 & 17483797 \\
605 & 15525080 \\
665 & 13788917 \\
731 & 10890052 \\
804 & 9669275 \\
884 & 8589857 \\
972 & 7631215 \\
1069 & 6776772 \\
1175 & 6020939 \\
1292 & 5347118 \\
1421 & 4747333 \\
1563 & 4214131 \\
1719 & 3741150 \\
\hline
\end{tabular}

\begin{tabular}{|c|c|c|c|}
\hline$L_{k}$ & $n_{k}$ & $L_{k}$ & $n_{k}$ \\
\hline 1890 & 3323382 & 7881 & 557957 \\
\hline 2079 & 2950258 & 8669 & 495180 \\
\hline 2286 & 2620862 & 9535 & 439662 \\
\hline 2514 & 2327160 & 10488 & 389839 \\
\hline 2765 & 2066024 & 11536 & 345918 \\
\hline 3041 & 1834523 & 12689 & 307046 \\
\hline 3345 & 1628901 & 13957 & 272420 \\
\hline 3679 & 1446024 & 15352 & 241798 \\
\hline 4046 & 1283655 & 16887 & 214746 \\
\hline 4450 & 1140213 & 18575 & 190486 \\
\hline 4895 & 1012659 & 20000 & 173506 \\
\hline 5384 & 898680 & & \\
\hline 5922 & 797641 & & \\
\hline 6514 & 708293 & & \\
\hline 7165 & 628813 & & \\
\hline
\end{tabular}

Values used for the fit: $L_{k}=1069 \ldots 20000$. CPU time 2064h. 


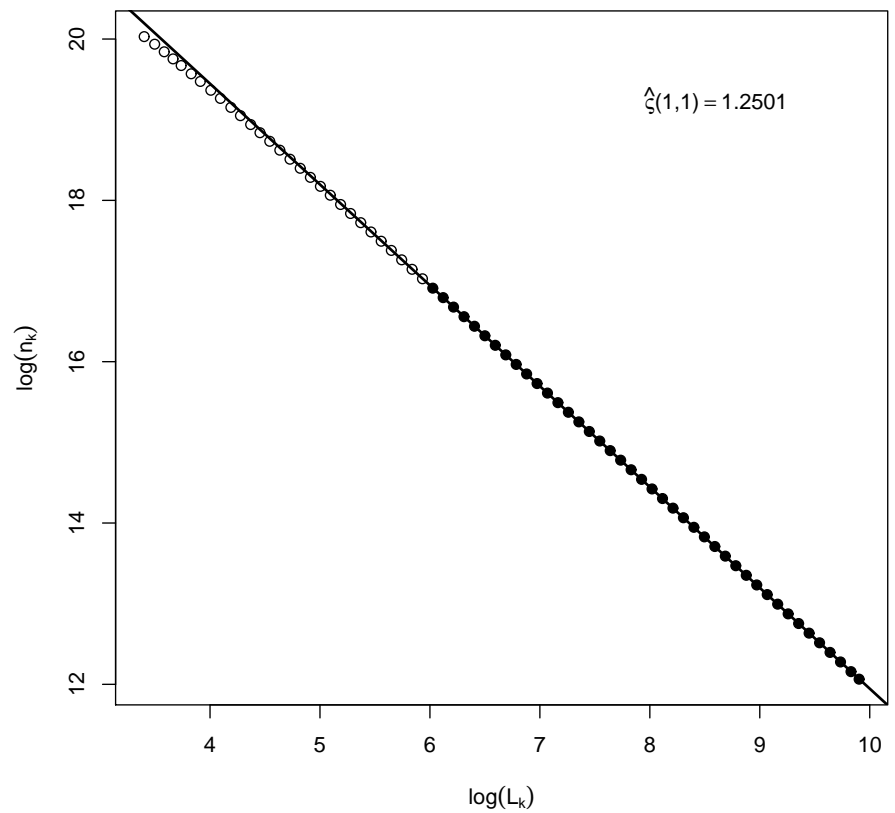

Figure 2: Linear regression for the simulation of $\varsigma_{2}(1,1)$.

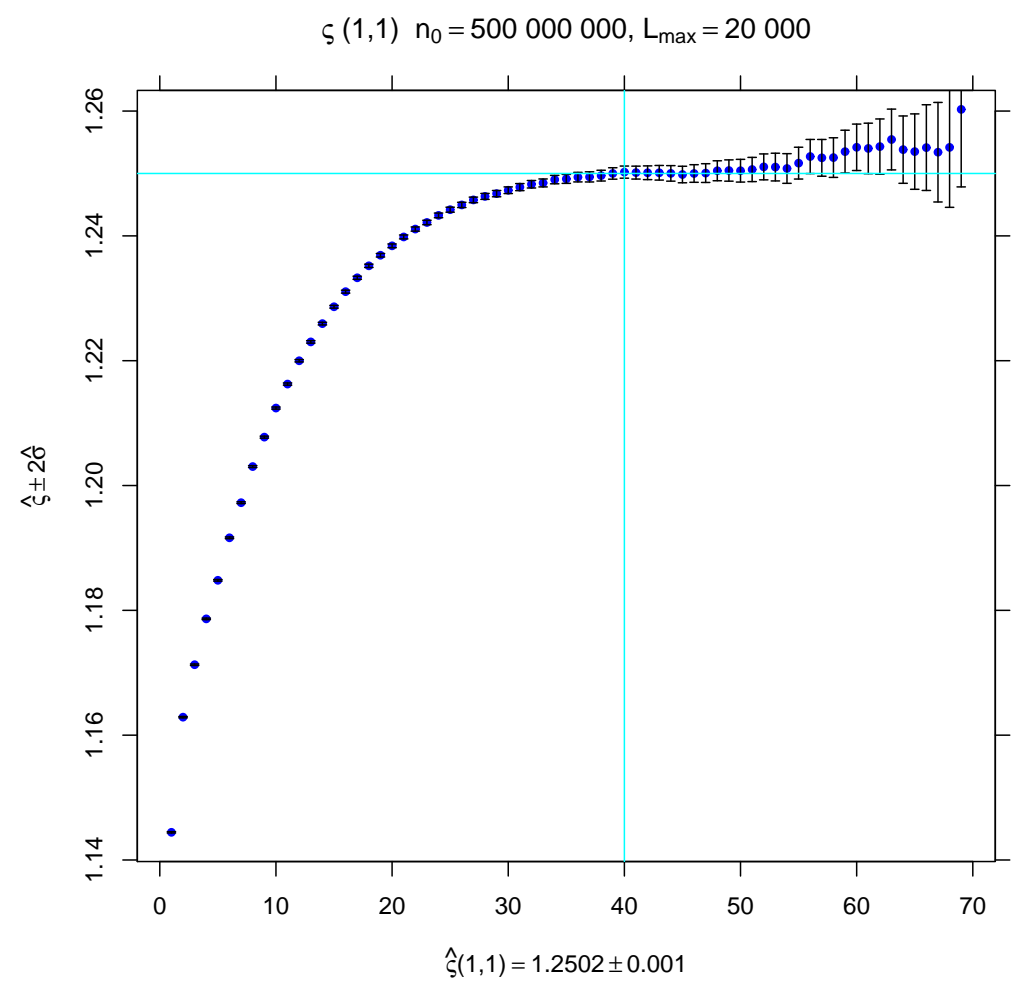

Figure 3: Simulation for $\varsigma_{2}(1,1)$. The co-ordinate shows $k_{\min }$, the ordinate shows the corresponding $\hat{\varsigma}$ with error bars. The vertical line indicates $k_{\min }=40$ which we chose for our estimate of $\hat{\varsigma}$. The horizontal line shows the true value. 


\subsubsection{Exponent $\varsigma(2,2)$.}

The exact value $\varsigma_{2}(2,2)=35 / 12=2.91666 \ldots$ is known. Also this simulation serves as a benchmark for our simulations.

\begin{tabular}{|r|r|}
\hline$L_{k}$ & $n_{k}$ \\
\hline 30 & 40000000000 \\
33 & 27956276949 \\
36 & 19934507109 \\
39 & 14769670878 \\
42 & 11270896745 \\
46 & 8156016609 \\
50 & 6108280379 \\
55 & 4423365460 \\
60 & 3315611015 \\
66 & 2432628801 \\
72 & 1842369408 \\
79 & 1375726309 \\
86 & 1056545535 \\
94 & 803537797 \\
103 & 607993657 \\
\hline
\end{tabular}

\begin{tabular}{|r|r|}
\hline$L_{k}$ & $n_{k}$ \\
\hline 113 & 459313243 \\
124 & 347384944 \\
136 & 263528000 \\
149 & 200799711 \\
163 & 153819037 \\
179 & 116600065 \\
196 & 89210485 \\
215 & 67932955 \\
236 & 51656232 \\
259 & 39313221 \\
284 & 30007400 \\
312 & 22780638 \\
343 & 17265563 \\
377 & 13094893 \\
414 & 9961095 \\
\hline
\end{tabular}

\begin{tabular}{|r|r|}
\hline$L_{k}$ & $n_{k}$ \\
\hline 455 & 7559087 \\
500 & 5737717 \\
550 & 4343548 \\
605 & 3288311 \\
665 & 2496057 \\
731 & 1893876 \\
804 & 1434709 \\
884 & 1087314 \\
972 & 823685 \\
1069 & 624023 \\
1175 & 473832 \\
1292 & 359121 \\
1421 & 271557 \\
1563 & 205432 \\
1719 & 155585 \\
\hline
\end{tabular}

\begin{tabular}{|r|r|}
\hline$L_{k}$ & $n_{k}$ \\
\hline 1890 & 117893 \\
2079 & 89442 \\
2286 & 67757 \\
2514 & 51314 \\
2765 & 38803 \\
3041 & 29341 \\
3345 & 22363 \\
3679 & 16949 \\
4046 & 12813 \\
4450 & 9738 \\
4895 & 7339 \\
5384 & 5571 \\
5922 & 4218 \\
6514 & 3229 \\
7165 & 2477 \\
\hline
\end{tabular}

\begin{tabular}{|r|r|}
\hline$L_{k}$ & $n_{k}$ \\
\hline 7881 & 1872 \\
8669 & 1450 \\
9535 & 1108 \\
10488 & 853 \\
11536 & 650 \\
12689 & 479 \\
13957 & 348 \\
15352 & 266 \\
16887 & 193 \\
18575 & 151 \\
20000 & 123 \\
& \\
& \\
& \\
\end{tabular}

Values used for the fit: $L_{k}=605 \ldots 20000$. CPU time $1879 \mathrm{~h}$.

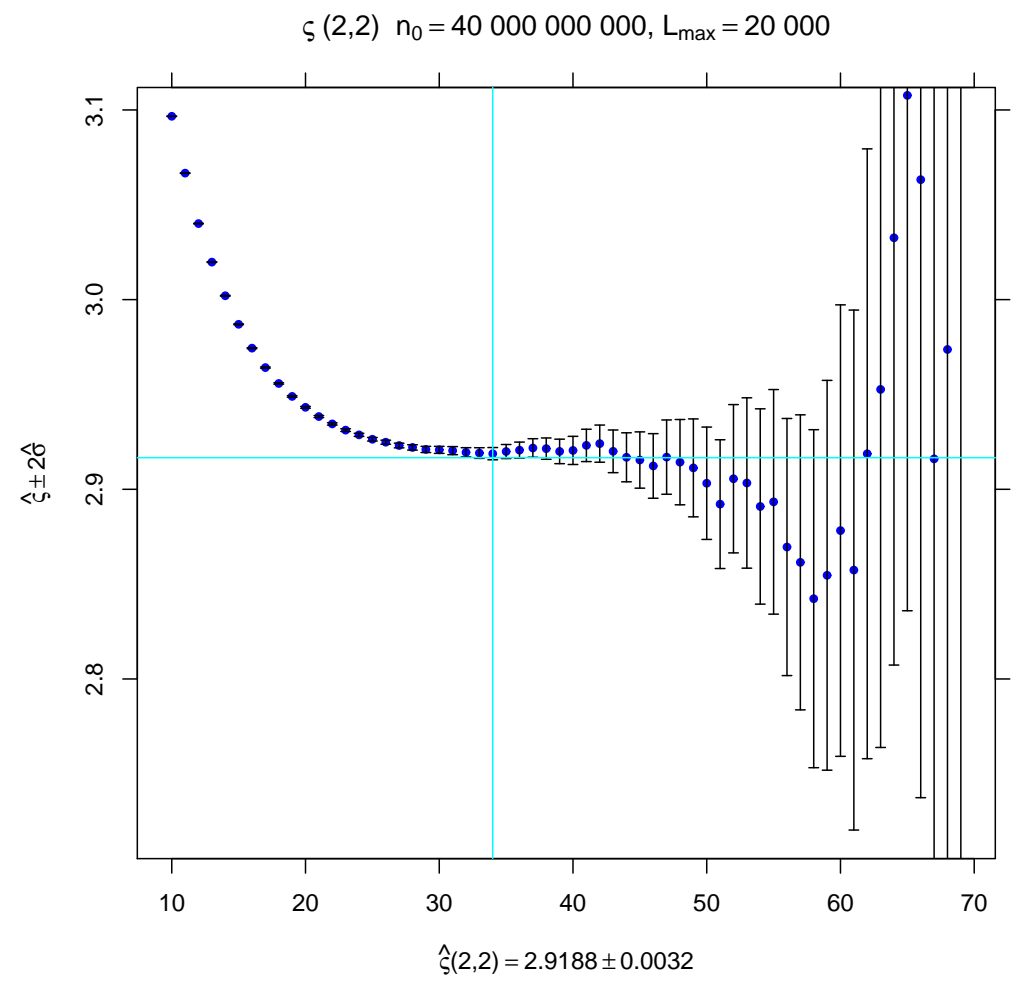

Figure 4: Simulation for $\varsigma_{2}(2,2)$. The co-ordinate shows $k_{\min }$, the ordinate shows the corresponding $\hat{\varsigma}$ with error bars. The vertical line indicates $k_{\min }=34$ which we chose for our estimate of $\hat{\varsigma}$. The horizontal line shows the true value. 


\subsubsection{Exponent $\varsigma_{3}(1,1,1)$.}

The exact value of $\varsigma_{3}(1,1,1)$ is unknown.

\begin{tabular}{|r|r|}
\hline$L_{k}$ & $n_{k}$ \\
\hline 30 & 60000000 \\
33 & 59710616 \\
36 & 58947709 \\
39 & 57896946 \\
42 & 56673833 \\
46 & 54898618 \\
50 & 53061195 \\
55 & 50777396 \\
60 & 48570208 \\
66 & 46070175 \\
72 & 43747356 \\
79 & 41266693 \\
86 & 39014779 \\
94 & 36696389 \\
103 & 34372986 \\
113 & 32097711 \\
124 & 29906035 \\
136 & 27820941 \\
\hline
\end{tabular}

\begin{tabular}{|r|r|}
\hline$L_{k}$ & $n_{k}$ \\
\hline 149 & 25859377 \\
163 & 24028791 \\
179 & 22226328 \\
196 & 20584233 \\
215 & 19009323 \\
236 & 17526913 \\
259 & 16147690 \\
284 & 14873454 \\
312 & 13666336 \\
343 & 12537025 \\
377 & 11494795 \\
414 & 10540910 \\
455 & 9652748 \\
500 & 8835893 \\
550 & 8076259 \\
605 & 7376857 \\
665 & 6740503 \\
731 & 6155608 \\
\hline
\end{tabular}

\begin{tabular}{|r|r|}
\hline$L_{k}$ & $n_{k}$ \\
\hline 804 & 5614962 \\
884 & 5121770 \\
972 & 4670981 \\
1069 & 4256241 \\
1175 & 3879722 \\
1292 & 3534606 \\
1421 & 3218775 \\
1563 & 2930010 \\
1719 & 2666485 \\
1890 & 2426899 \\
2079 & 2208165 \\
2286 & 2009516 \\
2514 & 1827541 \\
2765 & 1661614 \\
3041 & 1510468 \\
3345 & 1372149 \\
3679 & 1246493 \\
4046 & 1132343 \\
\hline
\end{tabular}

\begin{tabular}{|r|r|}
\hline$L_{k}$ & $n_{k}$ \\
\hline 4450 & 1028587 \\
4895 & 934379 \\
5384 & 848491 \\
5922 & 770449 \\
6514 & 699523 \\
7165 & 635064 \\
7881 & 576119 \\
8669 & 523319 \\
9535 & 474777 \\
10488 & 430885 \\
11536 & 391134 \\
12689 & 354964 \\
13957 & 321882 \\
15352 & 291736 \\
16887 & 264553 \\
18575 & 239803 \\
20432 & 217707 \\
22475 & 197364 \\
\hline
\end{tabular}

\begin{tabular}{|r|r|}
\hline$L_{k}$ & $n_{k}$ \\
\hline 24722 & 179046 \\
27194 & 162421 \\
29913 & 147273 \\
32904 & 133514 \\
36194 & 121333 \\
39813 & 109856 \\
43794 & 99544 \\
48173 & 90226 \\
52990 & 81910 \\
58289 & 74069 \\
64117 & 67148 \\
70528 & 60809 \\
77580 & 54981 \\
80000 & 53301 \\
& \\
& \\
& \\
\hline
\end{tabular}

Values used for the fit: $L_{k}=18575 \ldots 80000$. CPU time $8262 \mathrm{~h}$.

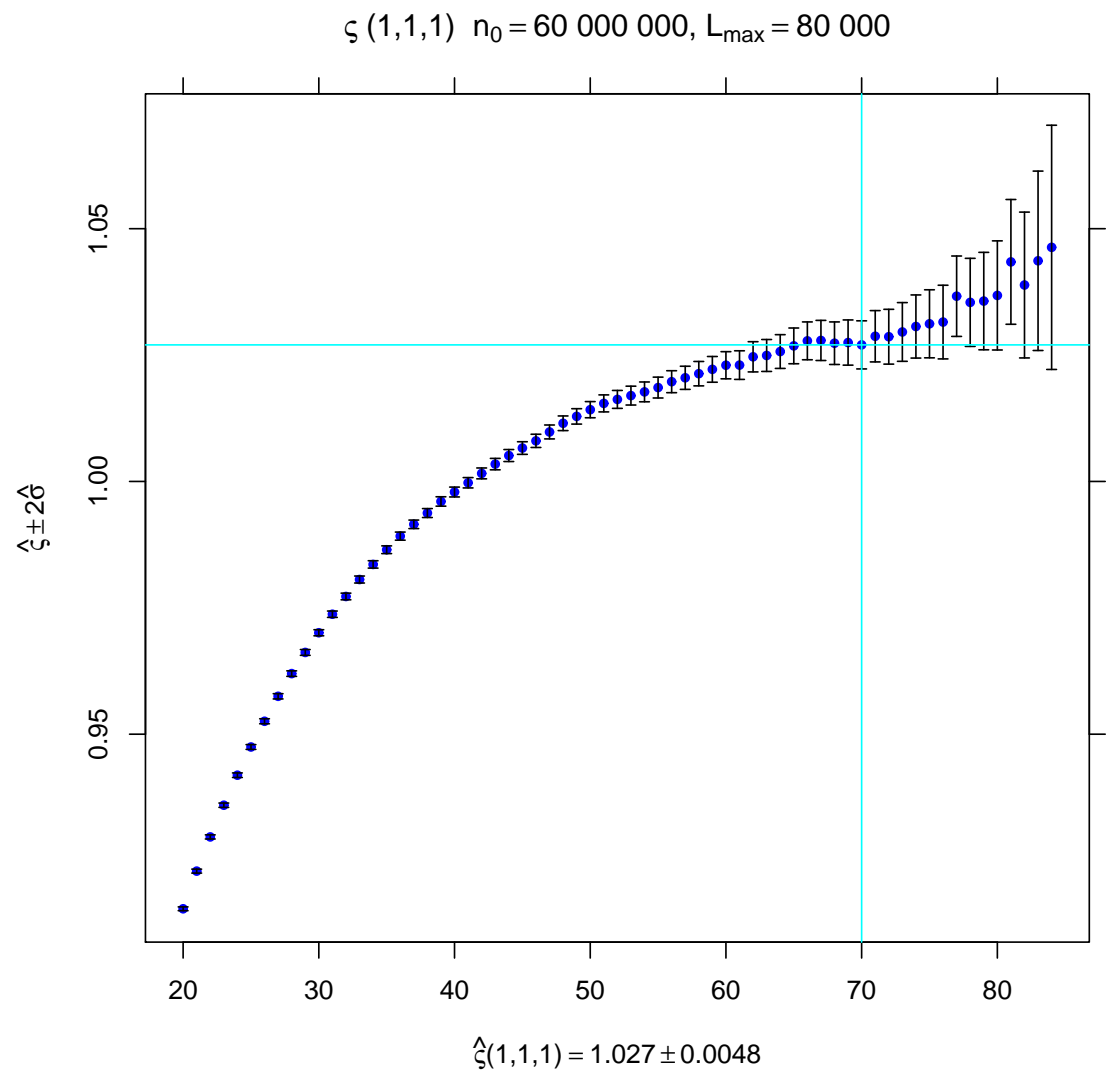

Figure 5: Simulation for $\varsigma_{3}(1,1,1)$. The co-ordinate shows $k_{\min }$, the ordinate shows the corresponding $\hat{\varsigma}$ with error bars. The vertical line indicates $k_{\min }=70$ which we chose for our estimate of $\hat{\varsigma}$. The horizontal line shows the estimated value. 


\subsubsection{Exponent $\varsigma_{3}(1,1,2)$.}

The exact value of $\varsigma_{3}(1,1,2)$ is unknown.

\begin{tabular}{|r|r|}
\hline$L_{k}$ & $n_{k}$ \\
\hline 30 & 200000000 \\
33 & 198136199 \\
36 & 193436538 \\
39 & 187242650 \\
42 & 180308394 \\
46 & 170682110 \\
50 & 161176218 \\
55 & 149901066 \\
60 & 139521065 \\
66 & 128301060 \\
72 & 118361321 \\
79 & 108205442 \\
86 & 99385254 \\
94 & 90671582 \\
103 & 82304628 \\
113 & 74445095 \\
124 & 67187208 \\
136 & 60566796 \\
\hline
\end{tabular}

\begin{tabular}{|r|r|}
\hline$L_{k}$ & $n_{k}$ \\
\hline 149 & 54585738 \\
163 & 49210675 \\
179 & 44119702 \\
196 & 39647836 \\
215 & 35523866 \\
236 & 31776899 \\
259 & 28415711 \\
284 & 25417521 \\
312 & 22668175 \\
343 & 20191522 \\
377 & 17981958 \\
414 & 16028002 \\
455 & 14267282 \\
500 & 12694594 \\
550 & 11279842 \\
605 & 10020648 \\
665 & 8909164 \\
731 & 7917614 \\
\hline
\end{tabular}

\begin{tabular}{|r|r|}
\hline$L_{k}$ & $n_{k}$ \\
\hline 804 & 7029965 \\
884 & 6245336 \\
972 & 5545792 \\
1069 & 4923405 \\
1175 & 4374033 \\
1292 & 3885012 \\
1421 & 3449618 \\
1563 & 3062025 \\
1719 & 2718548 \\
1890 & 2415286 \\
2079 & 2144282 \\
2286 & 1904636 \\
2514 & 1690316 \\
2765 & 1499756 \\
3041 & 1331441 \\
3345 & 1181425 \\
3679 & 1048523 \\
4046 & 930691 \\
\hline
\end{tabular}

\begin{tabular}{|r|r|}
\hline$L_{k}$ & $n_{k}$ \\
\hline 4450 & 826443 \\
4895 & 733837 \\
5384 & 651874 \\
5922 & 578486 \\
6514 & 513593 \\
7165 & 456239 \\
7881 & 405240 \\
8669 & 359523 \\
9535 & 319391 \\
10488 & 283792 \\
11536 & 251871 \\
12689 & 223767 \\
13957 & 198585 \\
15352 & 176146 \\
16887 & 156219 \\
18575 & 138744 \\
20432 & 123285 \\
22475 & 109424 \\
\hline
\end{tabular}

\begin{tabular}{|r|r|}
\hline$L_{k}$ & $n_{k}$ \\
\hline 24722 & 97235 \\
27194 & 86246 \\
29913 & 76472 \\
32904 & 67873 \\
36194 & 60371 \\
39813 & 53674 \\
43794 & 47628 \\
48173 & 42353 \\
52990 & 37627 \\
58289 & 33387 \\
64117 & 29608 \\
70528 & 26339 \\
77580 & 23407 \\
80000 & 22541 \\
& \\
& \\
& \\
\hline
\end{tabular}

Values used for the fit: $L_{k}=1069 \ldots 10000$. CPU time 5858h.

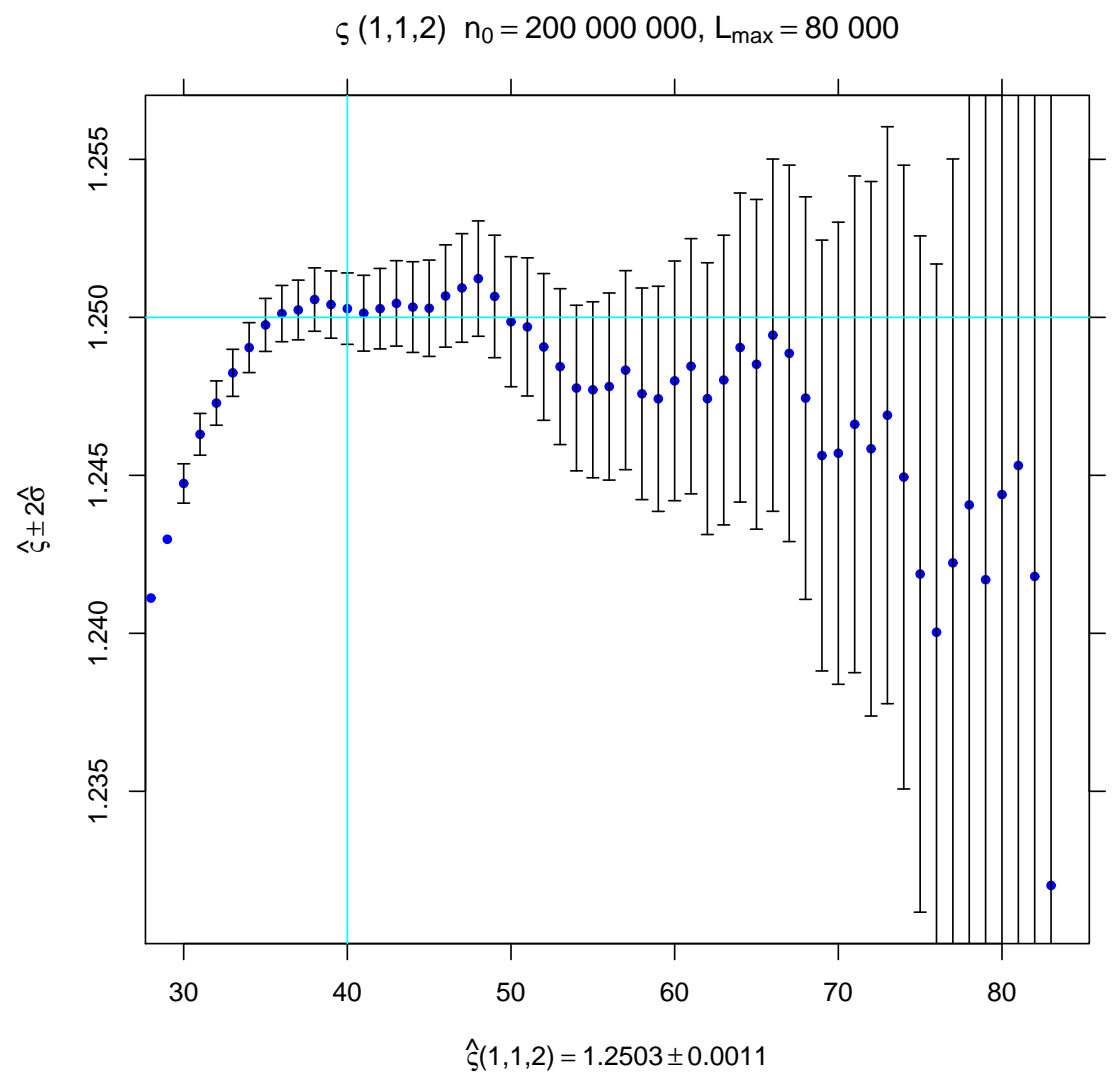

Figure 6: Simulation for $\varsigma_{3}(1,1,2)$. The co-ordinate shows $k_{\min }$, the ordinate shows the corresponding $\hat{\varsigma}$ with error bars. The vertical line indicates $k_{\min }=40$ which we chose for our estimate of $\hat{\varsigma}$. The horizontal line shows the conjectured value $\varsigma_{3}(1,1,2)=\varsigma_{2}(1,1)=5 / 4$. 


\subsubsection{Exponent $\varsigma_{4}(1,1,1,2)$.}

The exact value of $\varsigma_{4}(1,1,1,2)$ is unknown.

\begin{tabular}{|r|r|}
\hline$L_{k}$ & $n_{k}$ \\
\hline 30 & 200000000 \\
33 & 199921620 \\
36 & 199482984 \\
39 & 198565228 \\
42 & 197176986 \\
46 & 194683952 \\
50 & 191624492 \\
55 & 187237710 \\
60 & 182471375 \\
66 & 176514402 \\
72 & 170515344 \\
79 & 163642608 \\
86 & 157023575 \\
94 & 149860203 \\
103 & 142343356 \\
113 & 134666159 \\
\hline
\end{tabular}

\begin{tabular}{|r|r|}
\hline$L_{k}$ & $n_{k}$ \\
\hline 124 & 126992806 \\
136 & 119464557 \\
149 & 112181604 \\
163 & 105219661 \\
179 & 98203442 \\
196 & 91672706 \\
215 & 85304312 \\
236 & 79199999 \\
259 & 73434697 \\
284 & 68044645 \\
312 & 62863201 \\
343 & 57973159 \\
377 & 53412940 \\
414 & 49196512 \\
455 & 45240841 \\
500 & 41572496 \\
\hline
\end{tabular}

\begin{tabular}{|r|r|}
\hline$L_{k}$ & $n_{k}$ \\
\hline 550 & 38132997 \\
605 & 34954676 \\
665 & 32040238 \\
731 & 29348812 \\
804 & 26850724 \\
884 & 24560465 \\
972 & 22451887 \\
1069 & 20511329 \\
1175 & 18739197 \\
1292 & 17110256 \\
1421 & 15612645 \\
1563 & 14238301 \\
1719 & 12983348 \\
1890 & 11838167 \\
2079 & 10786022 \\
2286 & 9827571 \\
\hline
\end{tabular}

\begin{tabular}{|r|r|}
\hline$L_{k}$ & $n_{k}$ \\
\hline 2514 & 8950110 \\
2765 & 8149013 \\
3041 & 7419539 \\
3345 & 6752347 \\
3679 & 6144676 \\
4046 & 5589138 \\
4450 & 5082774 \\
4895 & 4621823 \\
5384 & 4201858 \\
5922 & 3819442 \\
6514 & 3470994 \\
7165 & 3155561 \\
7881 & 2866913 \\
8669 & 2604947 \\
9535 & 2366048 \\
10488 & 2149715 \\
\hline
\end{tabular}

\begin{tabular}{|r|r|}
\hline$L_{k}$ & $n_{k}$ \\
\hline 11536 & 1953289 \\
12689 & 1773558 \\
13957 & 1609927 \\
15352 & 1461067 \\
16887 & 1326172 \\
18575 & 1203707 \\
20432 & 1092672 \\
22475 & 991657 \\
24722 & 900187 \\
27194 & 816464 \\
29913 & 740704 \\
32904 & 672302 \\
36194 & 609756 \\
39813 & 553485 \\
40000 & 550828 \\
& \\
\hline
\end{tabular}

Values used for the fit: $L_{k}=27194, \ldots, 40000$. CPU time $35212 \mathrm{~h}$.

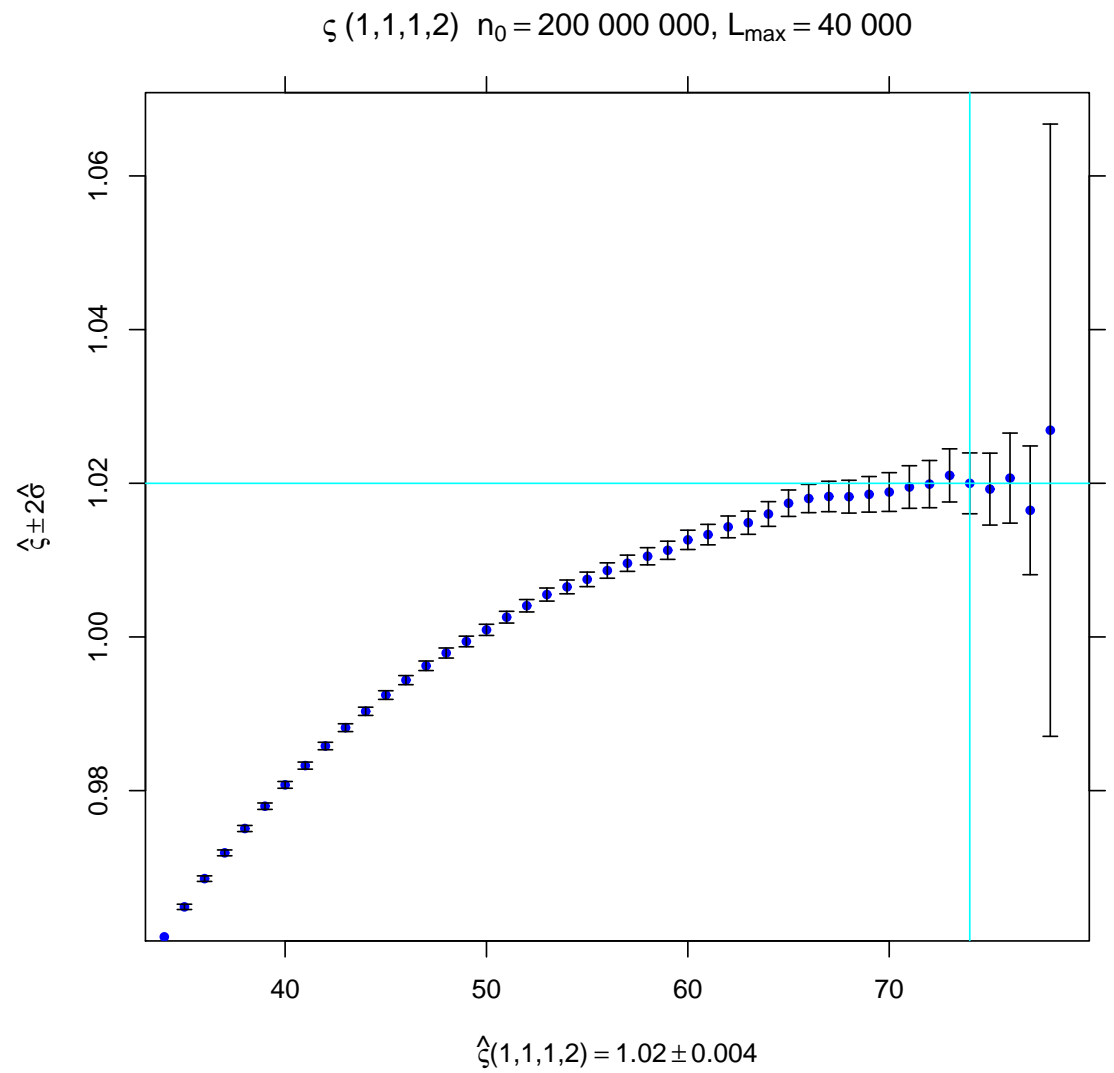

Figure 7: Simulation for $\varsigma_{4}(1,1,1,2)$. The co-ordinate shows $k_{\min }$, the ordinate shows the corresponding $\hat{\varsigma}$ with error bars. The vertical line indicates $k_{\min }=74$ which we chose for our estimate of $\hat{\varsigma}$. The horizontal line shows the estimated value. 
3.4.6 Exponent $\varsigma_{4}(1,1,1,1)$.

The exact value of $\varsigma_{4}(1,1,1,1)$ is unknown.

\begin{tabular}{|r|r|}
\hline$L_{k}$ & $n_{k}$ \\
\hline 30 & 20000000 \\
33 & 19996035 \\
36 & 19972855 \\
39 & 19923361 \\
42 & 19846358 \\
46 & 19704559 \\
50 & 19524417 \\
55 & 19258012 \\
60 & 18958581 \\
66 & 18573948 \\
72 & 18172653 \\
79 & 17698995 \\
86 & 17228707 \\
94 & 16704112 \\
103 & 16136193 \\
113 & 15537061 \\
124 & 14922694 \\
136 & 14299200 \\
\hline
\end{tabular}

\begin{tabular}{|r|r|}
\hline$L_{k}$ & $n_{k}$ \\
\hline 149 & 13677483 \\
163 & 13066040 \\
179 & 12433477 \\
196 & 11828110 \\
215 & 11220785 \\
236 & 10622843 \\
259 & 10040743 \\
284 & 9483483 \\
312 & 8935078 \\
343 & 8402393 \\
377 & 7892203 \\
414 & 7410458 \\
455 & 6946326 \\
500 & 6504855 \\
550 & 6081417 \\
605 & 5681289 \\
665 & 5304532 \\
731 & 4949662 \\
\hline
\end{tabular}

\begin{tabular}{|r|r|}
\hline$L_{k}$ & $n_{k}$ \\
\hline 804 & 4613179 \\
884 & 4297577 \\
972 & 4001670 \\
1069 & 3722426 \\
1175 & 3461745 \\
1292 & 3217081 \\
1421 & 2987877 \\
1563 & 2773980 \\
1719 & 2573328 \\
1890 & 2386906 \\
2079 & 2213006 \\
2286 & 2051046 \\
2514 & 1899964 \\
2765 & 1758768 \\
3041 & 1628269 \\
3345 & 1506688 \\
3679 & 1393480 \\
4046 & 1289159 \\
\hline
\end{tabular}

\begin{tabular}{|r|r|}
\hline$L_{k}$ & $n_{k}$ \\
\hline 4450 & 1191616 \\
4895 & 1101052 \\
5384 & 1017194 \\
5922 & 939586 \\
6514 & 867259 \\
7165 & 801006 \\
7881 & 739646 \\
8669 & 682392 \\
9535 & 629677 \\
10488 & 580582 \\
11536 & 535316 \\
12689 & 493266 \\
13957 & 454757 \\
15352 & 419131 \\
16887 & 386266 \\
18575 & 356146 \\
20432 & 328229 \\
22475 & 302420 \\
\hline
\end{tabular}

\begin{tabular}{|r|r|}
\hline$L_{k}$ & $n_{k}$ \\
\hline 24722 & 278878 \\
27194 & 256535 \\
29913 & 236157 \\
32904 & 217434 \\
36194 & 200369 \\
39813 & 184289 \\
43794 & 169567 \\
48173 & 156118 \\
52990 & 143655 \\
58289 & 132091 \\
64117 & 121391 \\
70528 & 111643 \\
77580 & 102601 \\
80000 & 99860 \\
& \\
& \\
& \\
\hline
\end{tabular}

Values used for the fit: $L_{k}=39813 \ldots 80000$. CPU time $18262 \mathrm{~h}$.

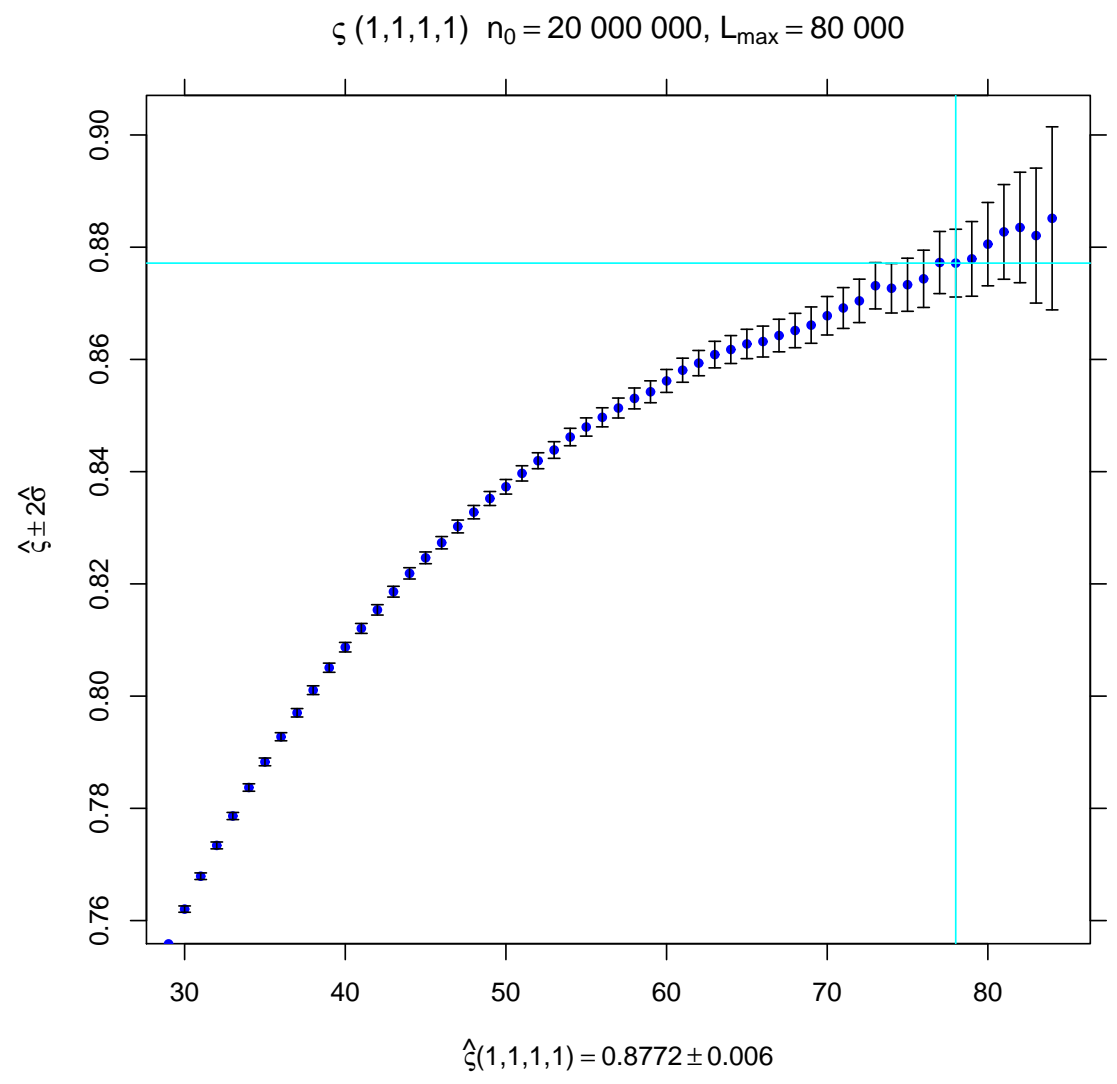

Figure 8: Simulation for $\varsigma_{4}(1,1,1,1)$. The co-ordinate shows $k_{\min }$, the ordinate shows the corresponding $\hat{\varsigma}$ with error bars. The vertical line indicates $k_{\min }=78$ which we chose for our estimate of $\hat{\varsigma}$. The horizontal line shows the estimated value. 


\subsubsection{Exponent $\varsigma_{5}(1,1,1,1,1)$.}

The exact value of $\varsigma_{5}(1,1,1,1,1)$ is unknown.

\begin{tabular}{|r|r|}
\hline$L_{k}$ & $n_{k}$ \\
\hline 30 & 744165 \\
33 & 744158 \\
36 & 744107 \\
39 & 743886 \\
42 & 743487 \\
46 & 742538 \\
50 & 741155 \\
55 & 738765 \\
60 & 735484 \\
66 & 730711 \\
72 & 725183 \\
79 & 718008 \\
86 & 710226 \\
94 & 700782 \\
103 & 689769 \\
113 & 677384 \\
\hline
\end{tabular}

\begin{tabular}{|r|r|}
\hline$L_{k}$ & $n_{k}$ \\
\hline 124 & 663493 \\
136 & 648801 \\
149 & 633473 \\
163 & 617246 \\
179 & 599664 \\
196 & 581885 \\
215 & 562961 \\
236 & 543820 \\
259 & 524462 \\
284 & 505282 \\
312 & 485535 \\
343 & 465955 \\
377 & 446300 \\
414 & 426779 \\
455 & 407418 \\
500 & 388692 \\
\hline
\end{tabular}

\begin{tabular}{|r|r|}
\hline$L_{k}$ & $n_{k}$ \\
\hline 550 & 370165 \\
605 & 352289 \\
665 & 335283 \\
731 & 318821 \\
804 & 302365 \\
884 & 286416 \\
972 & 271268 \\
1069 & 256593 \\
1175 & 242487 \\
1292 & 229014 \\
1421 & 216285 \\
1563 & 204002 \\
1719 & 192310 \\
1890 & 181055 \\
2079 & 170456 \\
2286 & 160399 \\
\hline
\end{tabular}

\begin{tabular}{|r|r|}
\hline$L_{k}$ & $n_{k}$ \\
\hline 2514 & 150828 \\
2765 & 141592 \\
3041 & 133025 \\
3345 & 124845 \\
3679 & 117257 \\
4046 & 109961 \\
4450 & 102996 \\
4895 & 96520 \\
5384 & 90434 \\
5922 & 84699 \\
6514 & 79371 \\
7165 & 74357 \\
7881 & 69601 \\
8669 & 65234 \\
9535 & 61002 \\
10488 & 56977 \\
\hline
\end{tabular}

\begin{tabular}{|r|r|}
\hline$L_{k}$ & $n_{k}$ \\
\hline 11536 & 53144 \\
12689 & 49724 \\
13957 & 46458 \\
15352 & 43355 \\
16887 & 40574 \\
18575 & 37983 \\
20432 & 35448 \\
22475 & 33083 \\
24722 & 30843 \\
27194 & 28666 \\
29913 & 26699 \\
32904 & 24955 \\
36194 & 23207 \\
39813 & 21616 \\
40000 & 21535 \\
\hline
\end{tabular}

Values used for the fit: $L_{k}=27194 \ldots 40000$. CPU time $1147 \mathrm{~h}$.

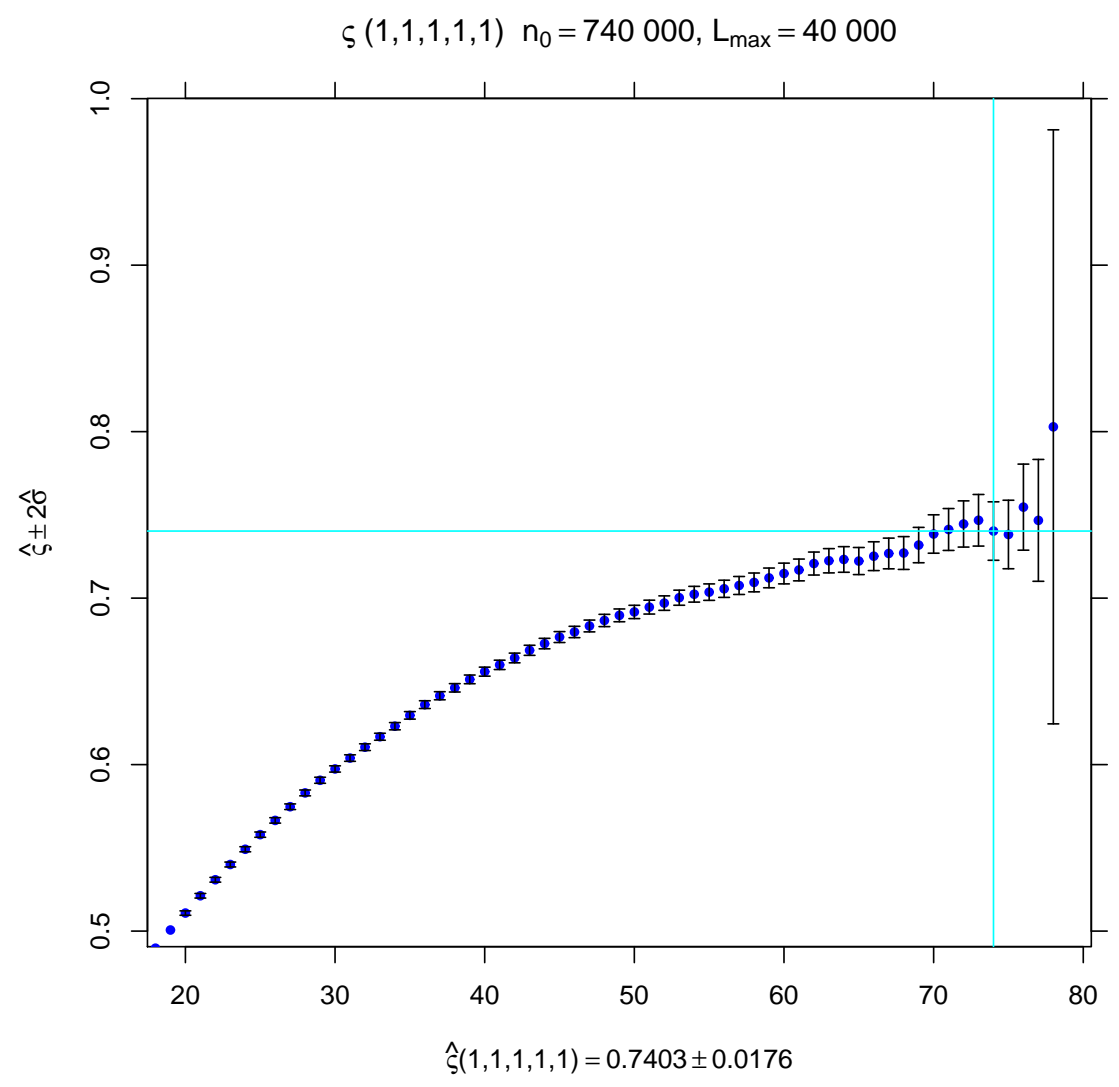

Figure 9: Simulation for $\varsigma_{5}(1,1,1,1,1)$. The co-ordinate shows $k_{\min }$, the ordinate shows the corresponding $\hat{\varsigma}$ with error bars. The vertical line indicates $k_{\min }=74$ which we chose for our estimate of $\hat{\varsigma}$. The horizontal line shows the estimated value. 


\subsubsection{Exponent $\varsigma_{3}(1,3,3)$.}

The exact value of $\varsigma_{3}(1,3,3)$ is unknown. As it turns out that $\varsigma_{3}(1,3,3)>2$, we have performed simulations according to our scheme 2 . That is, we have generated $n$ master samples of random walk paths that reach the boundary of the $L_{1}$-box (here $L_{1}=10000$ ). For each such master sample $i$ we have run $m=1000$ trials and have counted the fraction $\hat{p}_{i}$ of trials where the paths reached the boundary of the $L_{2}$-box (with $L_{2}=2 L_{1}$ ). As $n=18100$ we cannot give the complete data set $p_{1}, \ldots, p_{n}$ but rather give the empirical mean and the standard deviation of $\hat{p}$

$$
\hat{p}=0.155202983425414, \quad \hat{\sigma}_{p}=0.000536918044881792 .
$$

From this we compute

$$
\hat{\varsigma}_{3}(1,3,3)=2.6877718045551
$$

with standard deviation

$$
\hat{\sigma}=0.00499094143436367 \text {. }
$$

We conjecture that

$$
\varsigma_{3}(1,3,3)=\varsigma_{2}(1,3)=\frac{13+\sqrt{73}}{8}=2.693000 \ldots
$$

We conclude with a histogram of the values $p_{i}$ :

\section{$(1,3,3)$ Histogram of $p, 18100$ samples}

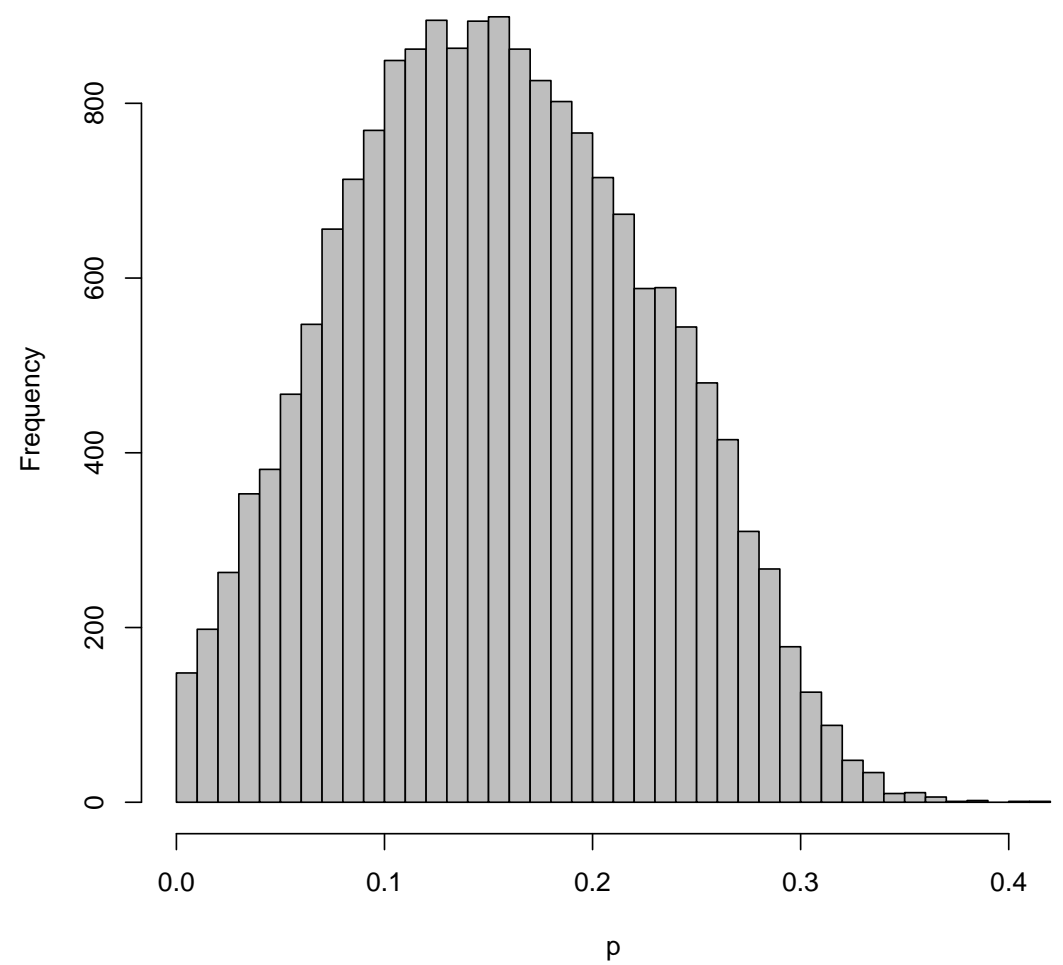

Figure 10: Histogram of the values $p_{i}$ for $\varsigma_{3}(1,3,3)$. 


\subsubsection{Exponent $\varsigma_{3}(2,2,2)$.}

The exact value of $\varsigma_{3}(2,2,2)$ is unknown. We have performed a simulation with the second scheme with $N=16000, n=1000, L_{1}=10000, L_{2}=20000$. Mean and standard deviation are

$$
\hat{p}=0.1449495, \quad \hat{\sigma}_{p}=0.000497221297799643 .
$$

From this we compute

$$
\hat{\varsigma}_{3}(2,2,2)=2.78637773802317
$$

with standard deviation

$$
\hat{\sigma}=0.00494888703003405 .
$$

\subsubsection{Exponent $\varsigma_{3}(2,2,3)$.}

The exact value of $\varsigma_{3}(2,2,3)$ is unknown. We conjecture

$$
\varsigma_{3}(2,2,3)=\varsigma_{2}(2,2)=\frac{35}{12}=2.916666 \ldots
$$

We have performed a simulation with the second scheme with $N=23000, n=1000, L_{1}=10000$, $L_{2}=20000$. Mean and standard deviation are

$$
\hat{p}=0.130559, \quad \hat{\sigma}_{p}=0.000444444142417374 .
$$

From this we compute

$$
\hat{\varsigma}_{3}(2,2,3)=2.93722618256156
$$

with standard deviation

$$
\hat{\sigma}=0.00491116935805033
$$

\subsubsection{Exponent $\varsigma_{3}(2,3,3)$.}

The exact value of $\varsigma_{3}(2,3,3)$ is unknown. We conjecture

$$
\varsigma_{3}(2,3,3)=\varsigma_{2}(2,3)=\frac{47+5 \sqrt{73}}{24}=3.738334113 \ldots
$$

We have performed a simulation with the second scheme with $N=1000, n=1000, L_{1}=10000$, $L_{2}=20000$. Mean and standard deviation are

$$
\hat{p}=0.073458, \quad \hat{\sigma}_{p}=0.00144828442088002 .
$$

From this we compute

$$
\hat{\varsigma}_{3}(2,3,3)=3.76693657262376
$$

with standard deviation

$$
\hat{\sigma}=0.0284439101500224 .
$$

This simulation was particularly time consuming (179543h CPU time) as the actual value of $\varsigma_{3}(2,3,3)$ is rather large and it thus takes a tremendous amount of time to generate each master sample. 


\subsubsection{Exponent $\varsigma_{4}(2,2,2,2)$.}

The exact value of $\varsigma_{4}(2,2,2,2)$ is unknown. We have performed a simulation with the second scheme with $N=16000, n=1000, L_{1}=10000, L_{2}=20000$. Mean and standard deviation are

$$
\hat{p}=0.157732125, \quad \hat{\sigma}_{p}=0.000521232849038418 .
$$

From this we compute

$$
\hat{\varsigma}_{4}(2,2,2,2)=2.66445157389522
$$

with standard deviation

$$
\hat{\sigma}=0.00476745017196814
$$

Acknowledgments: P.M. is supported by the Engineering and Physical Sciences Research Council (EPSRC) through an Advanced Research Fellowship.

\section{Bibliography}

[1] K. Burdzy, G. Lawler and T. Polaski. On the critical exponent for random walk intersections. J. Statist. Phys. 56, 1-12 (1989).

[2] F. Camia and C. M. Newman. Two-dimensional critical percolation: the full scaling limit. Comm. Math. Phys., 268, 1-38 (2006).

[3] F. Camia and C. M. Newman. Critical percolation exploration path and SLE 6 : a proof of convergence. Probab. Theory Related Fields 139, 473-519 (2007).

[4] J. Cardy. Critical percolation in finite geometries. J. Phys. A, 25, L201-L206 (1992).

[5] B. Duplantier. Random walks and quantum gravity in two dimensions. Phys. Rev. Lett., 81, 5489-5492 (1998).

[6] B. Duplantier and K.-H. Kwon. Conformal invariance and intersections of random walks. Phys. Rev. Lett., 61, 2514-2517 (1988).

[7] B. Duplantier and S. Sheffield. Duality and the Knizhnik-Polyakov-Zamolodchikov Relation in Liouville Quantum Gravity. Phys. Rev. Lett. 102, 150603 (2009).

[8] A. Klenke and P. Mörters. The multifractal spectrum of Brownian intersection local time. Ann. Probab. 33, 1255-1301 (2005).

[9] D. E. Knuth. The art of computer programming. Vol. 2, 3rd edition. Addison-Wesley, Boston MA (2005).

[10] G. F. LAwLER. Intersections of random walks with random sets. Israel J. Math. 65, 113-132 (1989).

[11] G. F. LAWLER. Intersections of random walks. Birkhäuser, Boston MA (1991).

[12] G. F. Lawler. Nonintersecting planar Brownian motions. Math. Phys. El. Journal, 1, Paper 4, pp 1-35 (1995).

[13] G. F. LAwLER. Hausdorff dimension of cut points for Brownian motion. El. Journal Probab., 1, Paper 2, pp 1-20 (1996).

[14] G. F. LAwler, O. Schramm and W. Werner. Values of Brownian intersection exponents I: Half-plane exponents. Acta Math., 187, 237-273 (2001).

[15] G. F. Lawler, O. Schramm and W. Werner. Values of Brownian intersection exponents II: Plane exponents. Acta Math., 187, 275-308 (2001).

[16] G. F. Lawler, O. Schramm and W. Werner. Values of Brownian intersection exponents III: Two-sided exponents. Ann. Inst. Henri Poincaré, 38, 109-123 (2002).

[17] G. F. Lawler, O. Schramm and W. Werner. Analyticity of intersection exponents for planar Brownian motion. Acta Math. 189, 179-201 (2002).

[18] P. L. Leath and G R Reich. Scaling form for percolation cluster sizes and perimeters. J. Phys. C 11, 4017-4036 (1978). 
[19] E. L. Lehmann. Theory of point estimation. Wiley, New York (1983).

[20] B. Li and A. SokAL. High-precision Monte Carlo test of the conformal-invariance predictions for two-dimensional mutually avoiding walks. J. Statist. Phys. 61, 723-748 (1990).

[21] P. Mörters and Y.Peres. Brownian motion. Cambridge University Press (2009).

[22] P. Mörters and N.-R. SHIEH. The exact packing measure of Brownian double points. Probab. Theory Related Fields 143, 113-136 (2009).

[23] R. Rhodes and V. VARGAs. KPZ formula for log-infinitely divisible multifractal random measures. arXiv:0807.1036 (July 2008).

[24] B. Saleur and B. Duplantier. Exact determination of the percolation hull exponent in two dimensions. Phys. Rev. Lett., 58, 2325 - 2328 (1987).

[25] S. Smirnov. Critical percolation in the plane: conformal invariance, Cardy's formula, scaling limits. C. R. Acad. Sci. Paris Sr. I Math. 333, 239-244 (2001).

[26] R.F. Voss. The fractal dimension of percolation cluster hulls. J. Phys. A 17, L373-L377 (1984). 